\title{
The demand-side grid (dsgrid) model
}

Elaine T. Hale, Ph.D.

September 6, 2018 


\section{$20^{\text {th }}$ century energy perspectives}

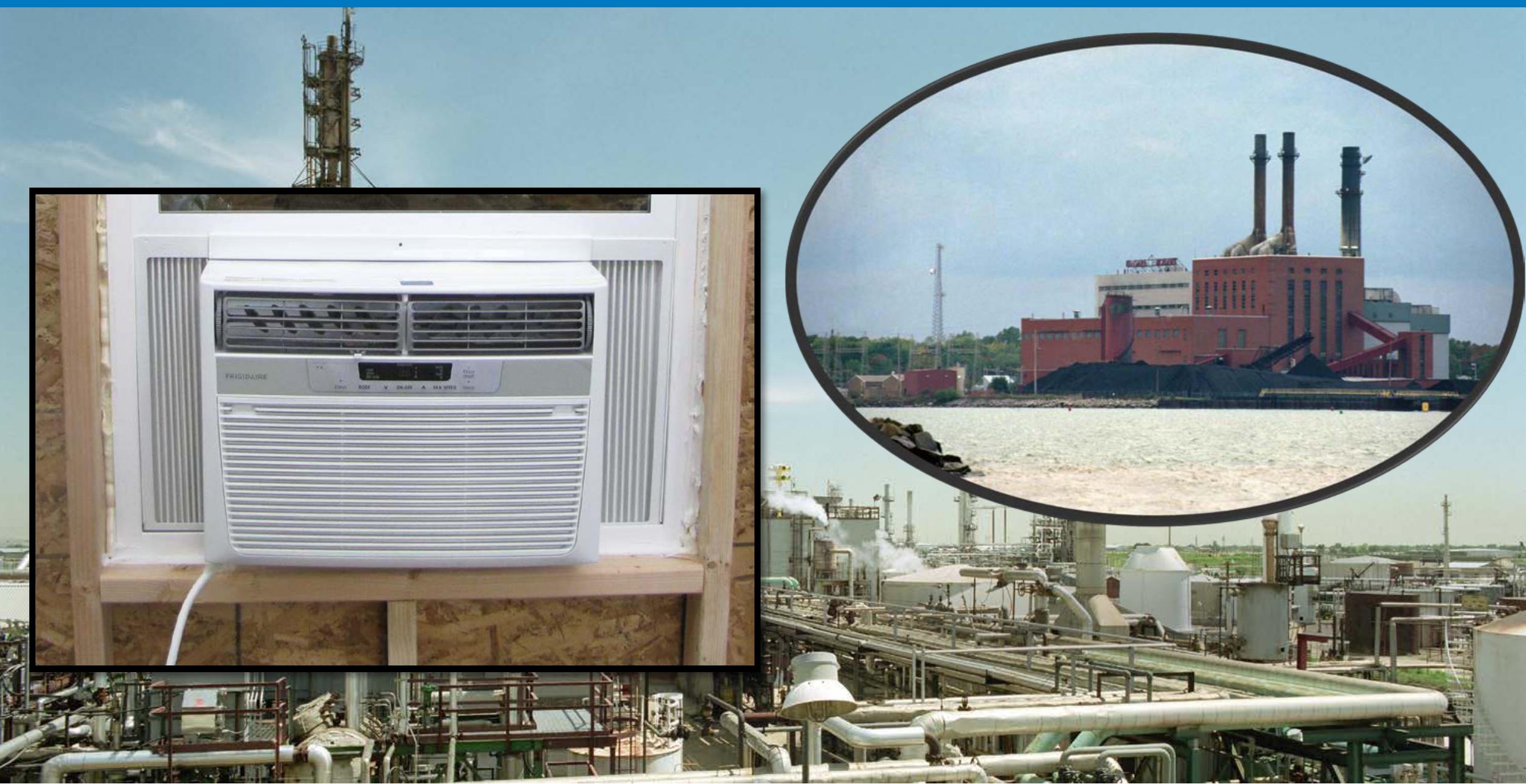



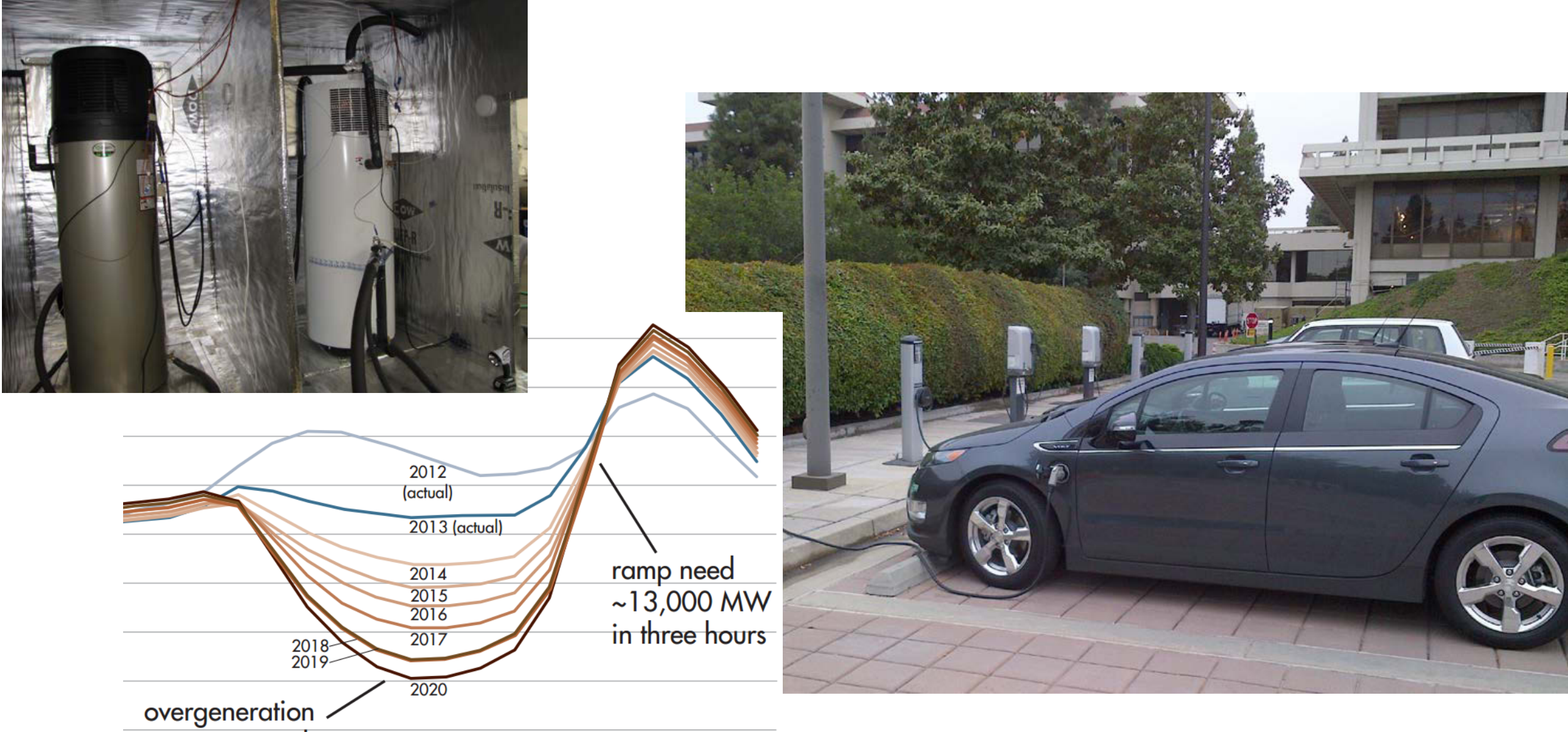

risk

Source: https://www.caiso.com/Documents/FlexibleResourcesHelpRenewables_FastFacts.pdf

\section{Emerging $21^{\text {st }}$ century perspectives}


The demand-side grid (dsgrid) model creates highly resolved timesynchronous load data by leveraging sector-specific modeling expertise

Bottom-up modeling of buildings, industry, and electric vehicles to enable:

- Future projections and whatif scenarios for load shape in addition to magnitude

- Realistic estimates of potential load flexibility (i.e., demand response)

- Understand interactions between energy efficiency and demand response potential (also renewables and DERs)

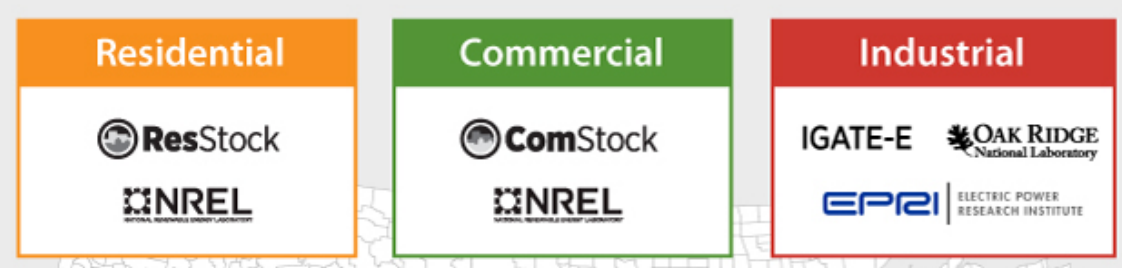

Transport
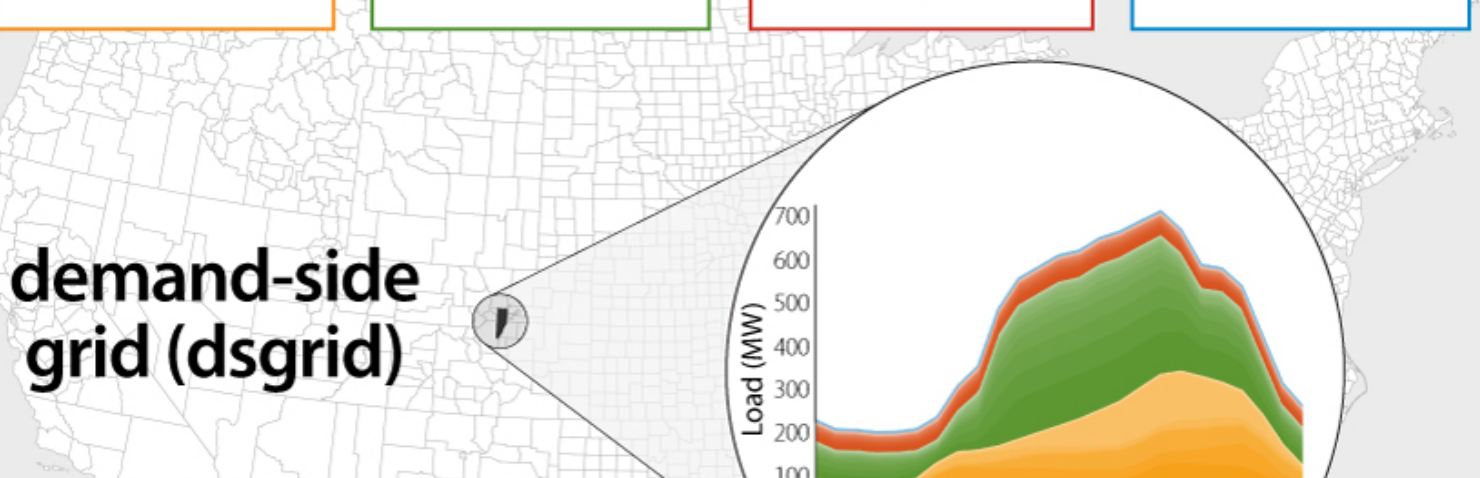

EVI-PRO GNREL 
Residential

;)ResStock

ONREL
Commercial

ComStock ONREL

\section{Industrial}

IGATE-E NOAK RIDGE

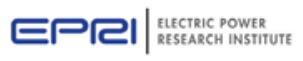

Transport

SE EVI-PRO

ONREL

dsgrid leverages decades of sectorspecific energy modeling

- High-quality modeling of each sector

- Breaks down energy-sector silos to enable cross-disciplinary understanding and holistic design

Buildings represent $\mathbf{7 1 \%}$ of U.S. electricity use, and building energy modeling is a particularly mature field

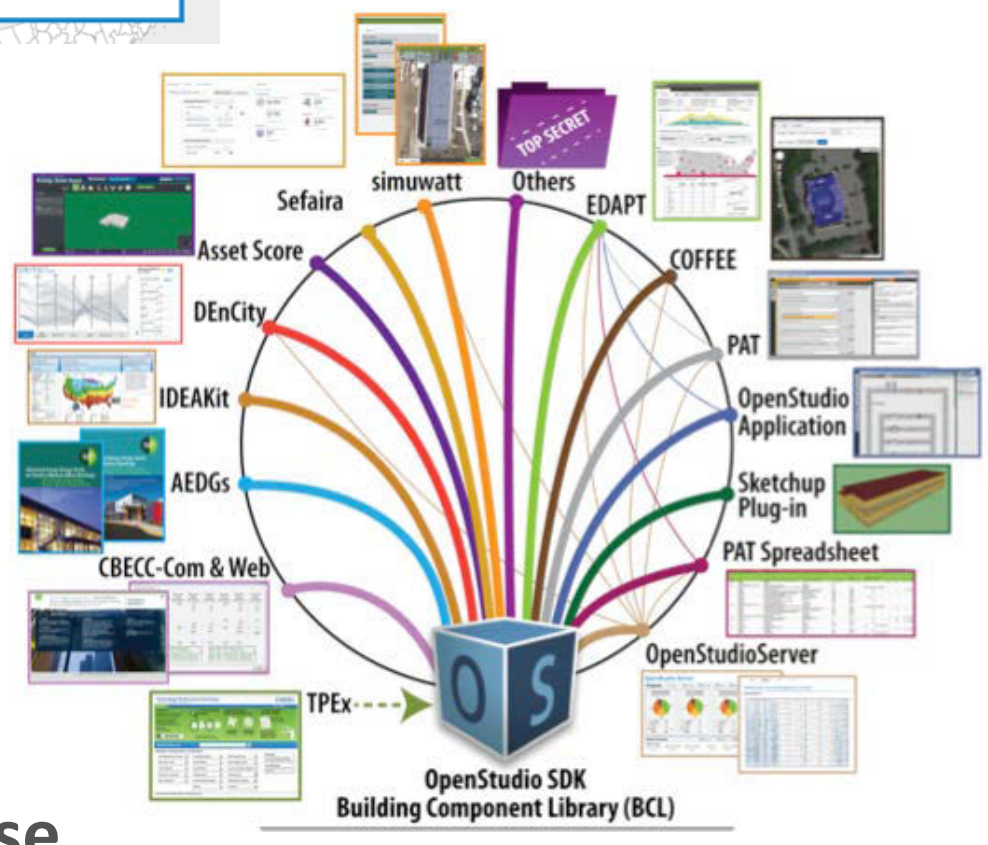




\section{Load models vary in extent, resolution, data, and methods}
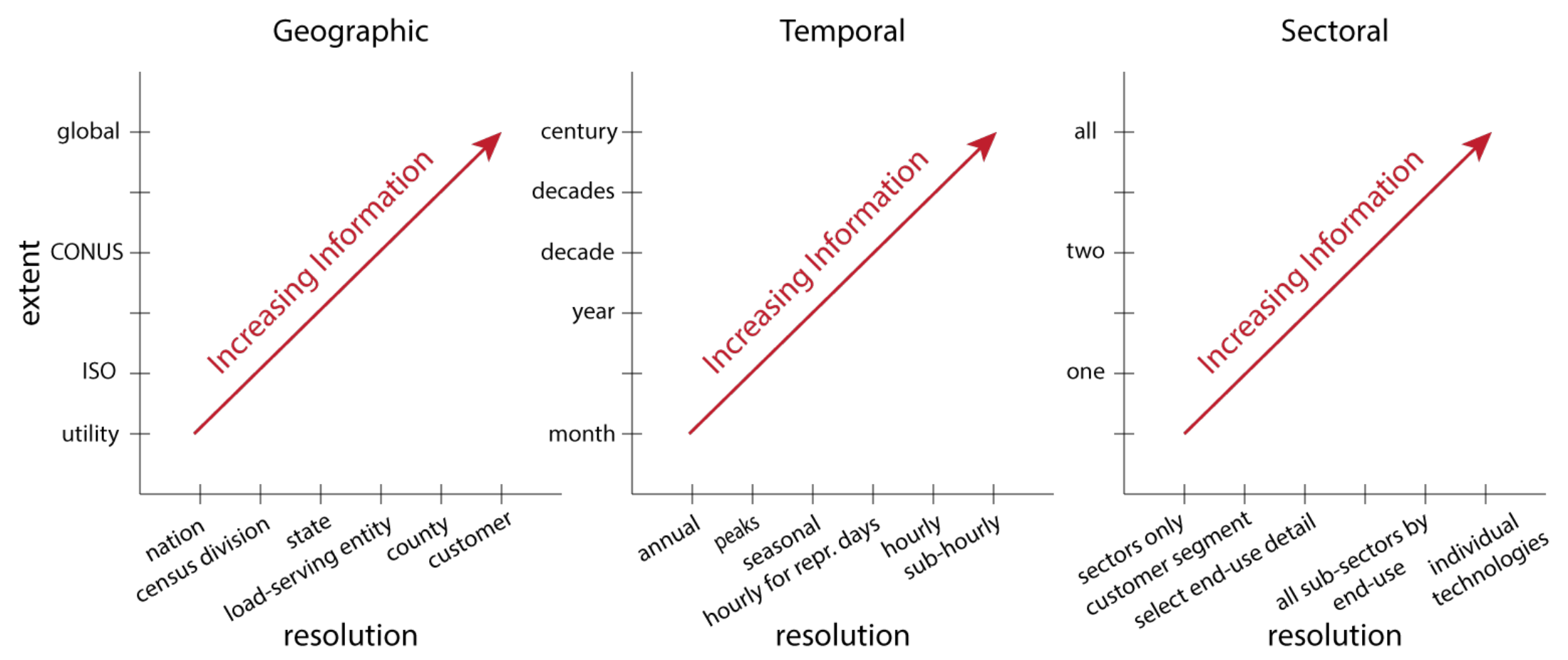


\section{large geographic and temporal extents}

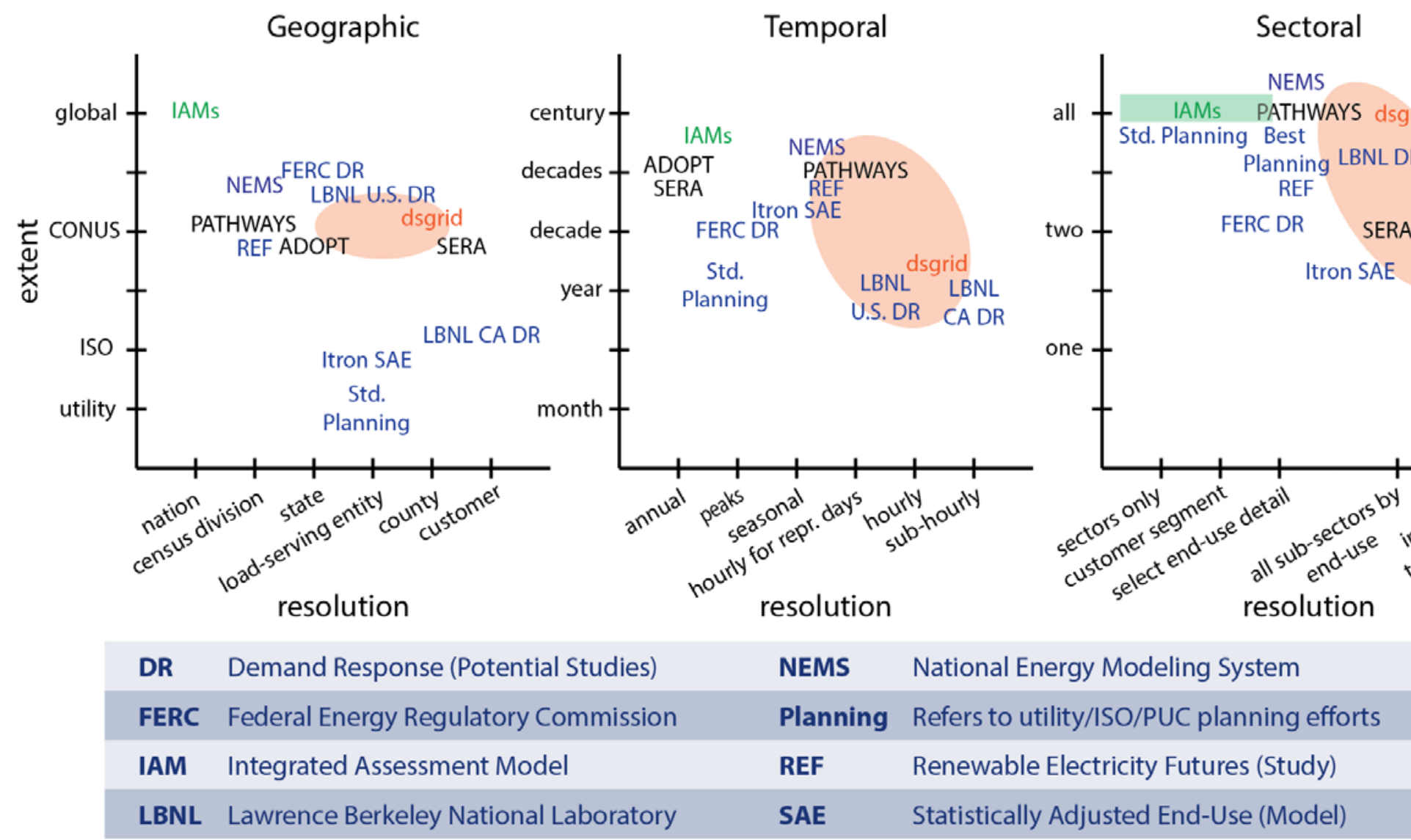




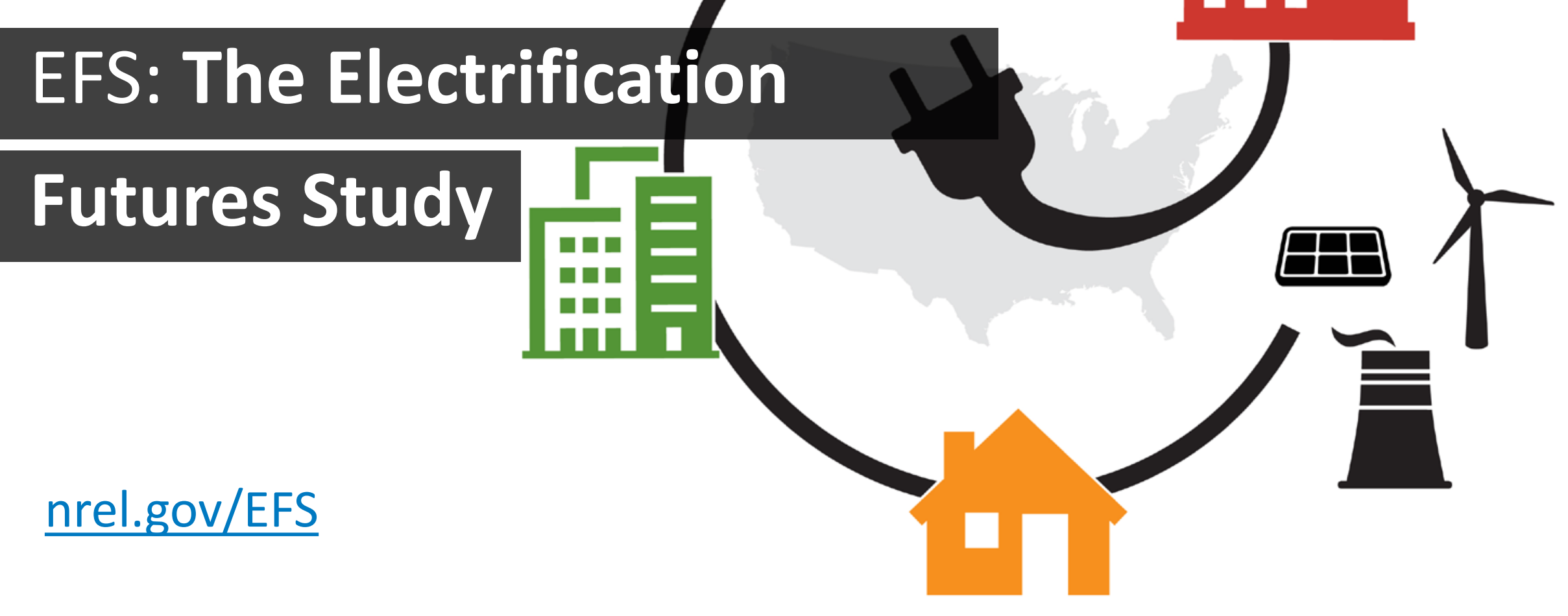


How do we plan for widespread electrification?

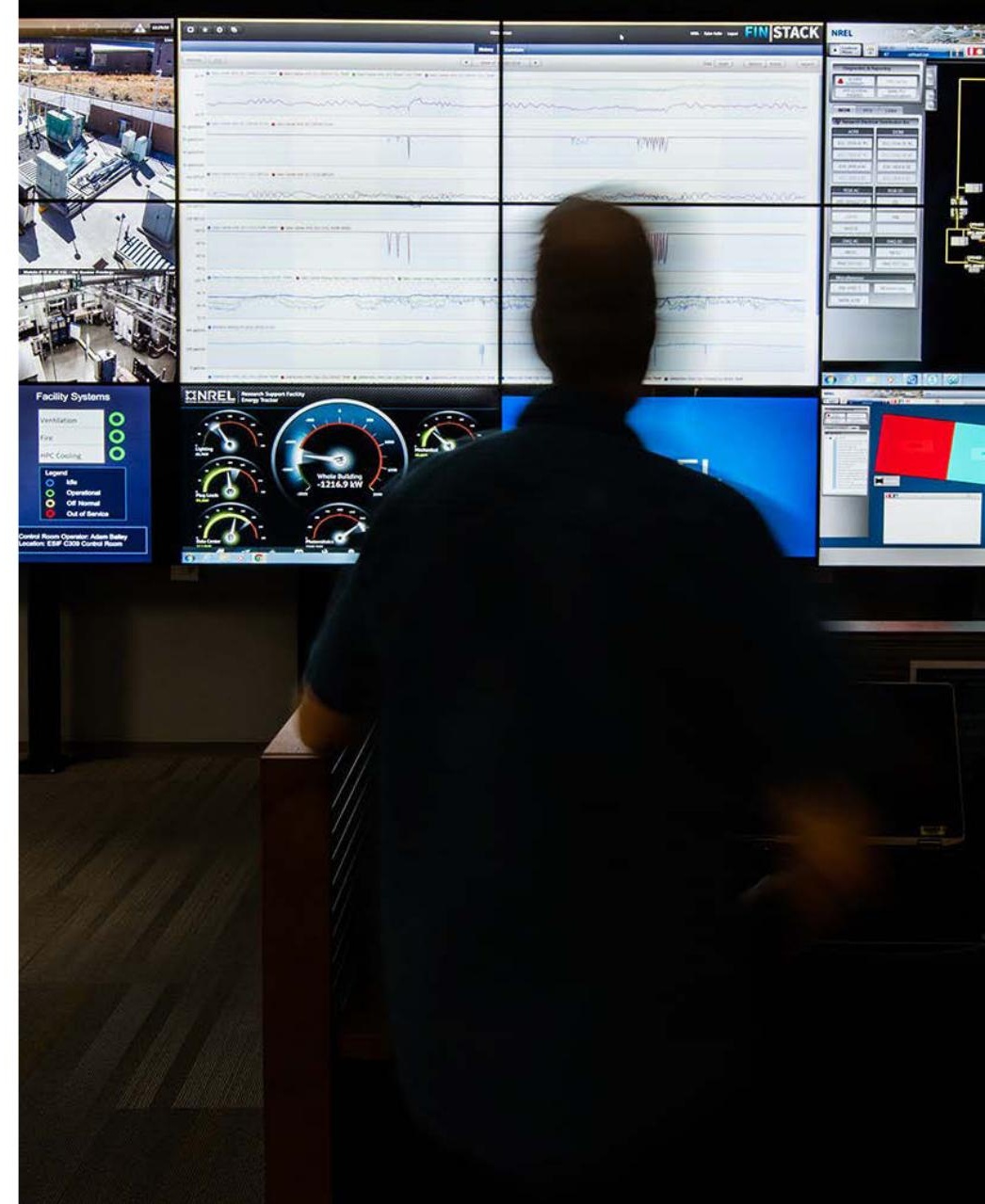




\section{Collaborators from:}

- EPRI

- Evolved Energy Research

- Northern Arizona University

- Oak Ridge National Laboratory

- Lawrence Berkeley National Laboratory

- U.S. Department of Energy

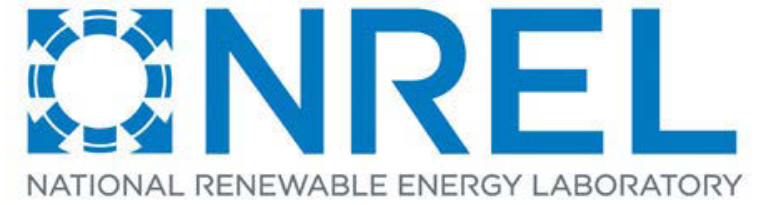

- Strategic Energy Analysis

- Transportation and Hydrogen Systems

- Buildings and Thermal Systems
Committee of 19 experts from industry and consultants, labs, government, NGOs 


\section{Answering crucial questions about:}

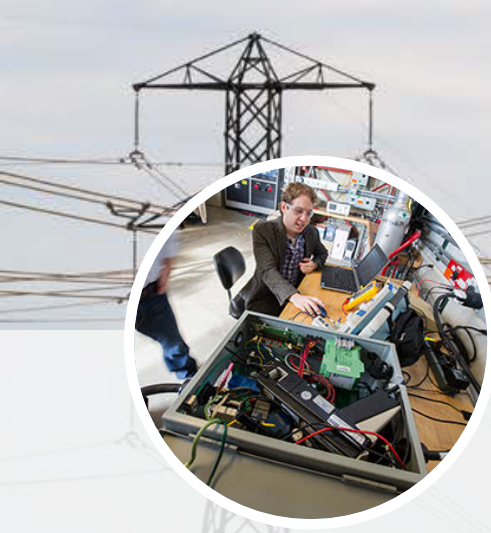

Technologies

What electric technologies are available now, and how might they advance?

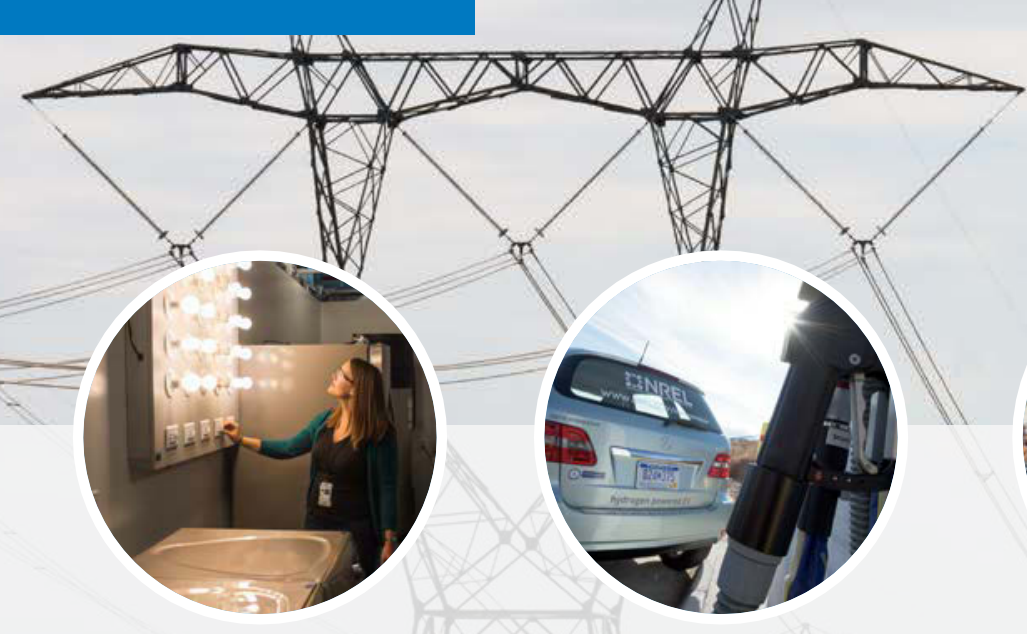

\section{Consumption}

How might electrification impact electricity demand and use patterns?

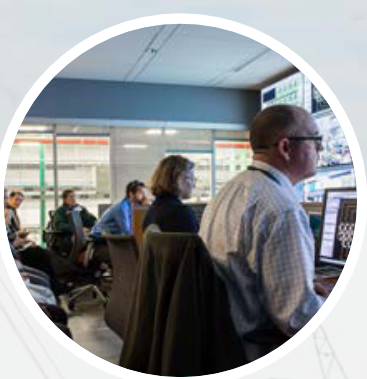

\section{Flexibility}

How would the electricity system need to transform to meet changes in demand?
What role might demand-side flexibility play to support reliable operations?

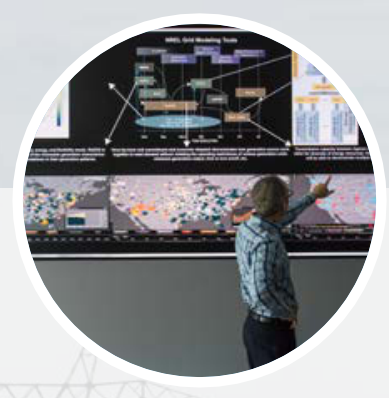

\section{Impacts}

What are the

potential costs, benefits, and impacts of widespread electrification? 


\section{Progress to date}

Technology cost and performance (December 2017)

Demand-side adoption scenarios (June 2018)

dsgrid model documentation (August 2018)

Supply-side evolution scenarios (2019)

Impacts of electrification (2019)

Electricity system operations ( 2020)

Value of demand-side flexibility ( 2020) 


\section{Historical Calibration Data Sources}
Planning
Region
Hourly
ISO / FERC 714 System Generation Data
Utility
by State
Utility
by State
Annual

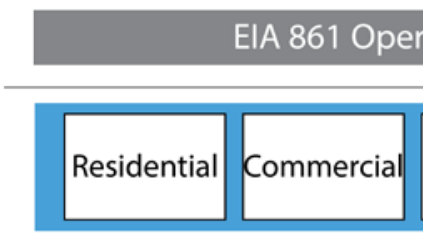
Annual
Residential
Industrial
Transport
EIA 861 Retail Sales \& Operational Data

Centralized Supply

\section{T \& D Losses}

\section{dsgrid model architecture}

\section{dsgrid Model Components}

\begin{tabular}{|c|c|c|c|c|c|c|c|c|}
\hline $\begin{array}{l}\text { State } \\
\text { (or County) }\end{array}$ & Hourly & \multicolumn{3}{|c|}{$\mathrm{CHP}$} & $\mathrm{CHP}$ & \multicolumn{2}{|r|}{$\begin{array}{l}\text { Distributed } \\
\text { Generation }\end{array}$} & \\
\hline $\begin{array}{l}\text { County } \\
\text { (per capita) }\end{array}$ & Hourly & $\begin{array}{r}\text { Muni } \\
\text { Water S }\end{array}$ & $\begin{array}{l}\text { ipal } \\
\text { rvices }\end{array}$ & $\begin{array}{l}\text { Outd } \\
\text { Light }\end{array}$ & & & $\begin{array}{c}\text { Non-Sectoral } \\
\text { Gap Models }\end{array}$ & \\
\hline $\begin{array}{l}\text { County } \\
\text { (or State) }\end{array}$ & Hourly & $\begin{array}{c}\text { Residential } \\
\text { Gaps }\end{array}$ & Comm & $\begin{array}{l}\text { rcial } \\
\text { s }\end{array}$ & $\begin{array}{l}\text { Industrial } \\
\text { Gaps }\end{array}$ & $\begin{array}{l}\text { Transport } \\
\text { Gaps }\end{array}$ & $\begin{array}{c}\text { Sectoral } \\
\text { Gap Models }\end{array}$ & Site-Level Demand \\
\hline County & Hourly & ResStock & Com & ock & IGATE-E & $\begin{array}{l}\text { SERA } \\
\text { / EVI-Pro }\end{array}$ & $\begin{array}{l}\text { Core, Detailed } \\
\text { Sector Models }\end{array}$ & \\
\hline Geographic & Temporal & Residential & Comr & ercial & Industrial & Transport & & \\
\hline
\end{tabular}




\section{dsgrid model documentation: methods and 2012 \\ U.S. electricity demand}

Hourly data for the contiguous United States (CONUS) for four representative weeks, aggregated by model component

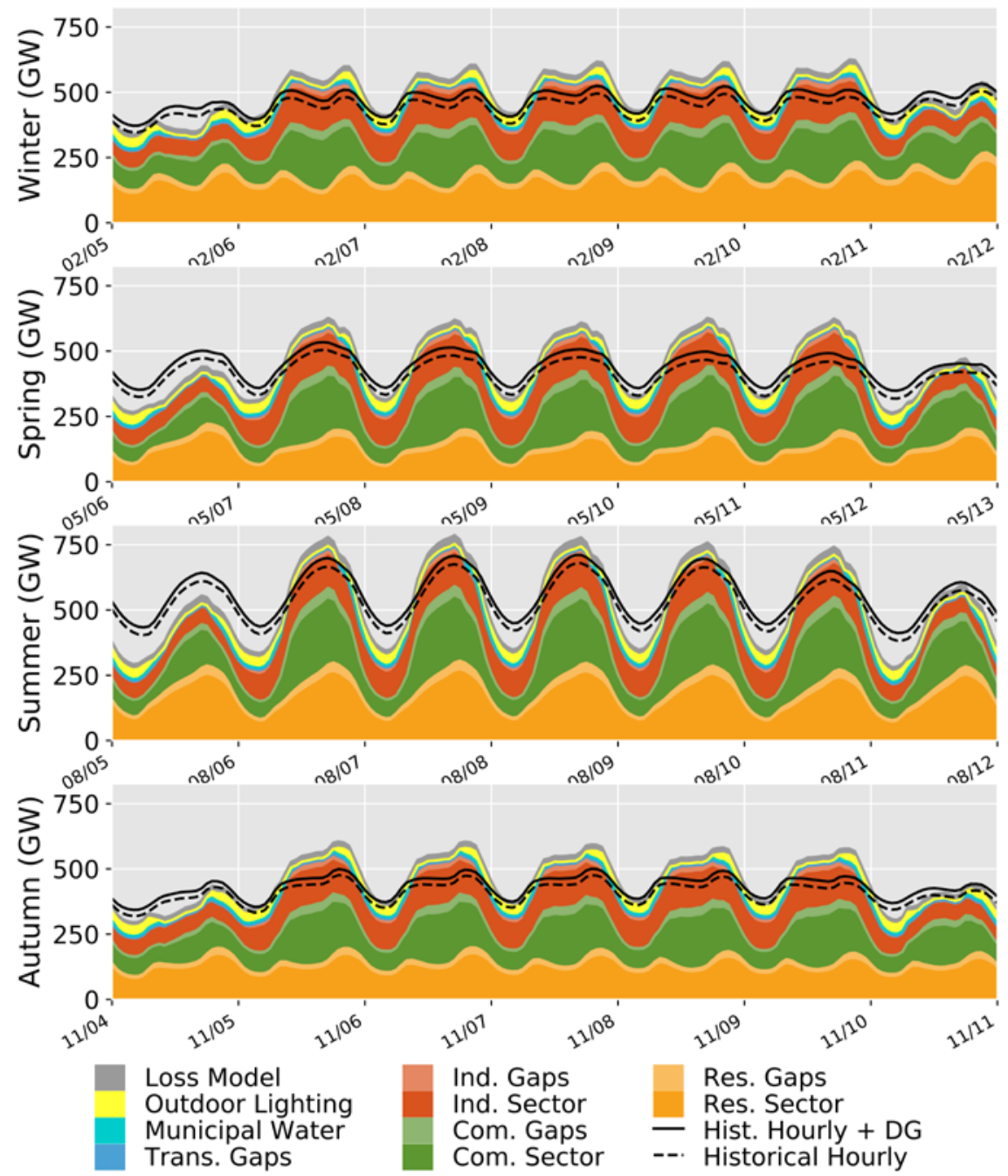




\section{Historical Calibration Data Sources}

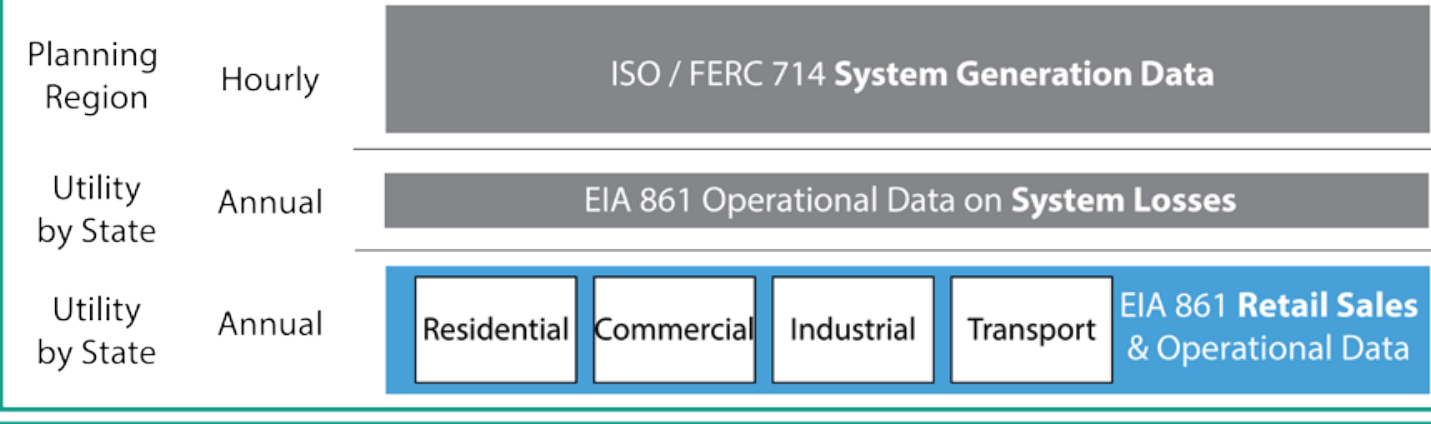

Centralized Supply

\section{T \& D Losses}

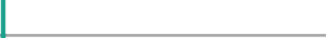

Site-Level Supply

\section{dsgrid Model Components}

\begin{tabular}{|c|c|c|c|c|c|c|c|}
\hline $\begin{array}{l}\text { State } \\
\text { (or County) }\end{array}$ & Hourly & $\frac{\mathrm{CHP}}{\mathrm{DPV}}$ & $\frac{\mathrm{CH}}{\mathrm{DF}}$ & & $\frac{\mathrm{CHP}}{\mathrm{DPV}}$ & & $\begin{array}{l}\text { Distributed } \\
\text { Generation }\end{array}$ \\
\hline $\begin{array}{l}\text { County } \\
\text { (per capita) }\end{array}$ & Hourly & $\begin{array}{r}\text { Munic } \\
\text { Water Se }\end{array}$ & ervices & $\begin{array}{l}\text { Outc } \\
\text { Ligh }\end{array}$ & & & $\begin{array}{c}\text { Non-Sectoral } \\
\text { Gap Models }\end{array}$ \\
\hline $\begin{array}{l}\text { County } \\
\text { (or State) }\end{array}$ & Hourly & $\begin{array}{l}\text { Residential } \\
\text { Gaps }\end{array}$ & $\begin{array}{r}\text { Comm } \\
\mathrm{Ga}\end{array}$ & ercial & $\begin{array}{l}\text { Industrial } \\
\text { Gaps }\end{array}$ & $\begin{array}{c}\text { Transport } \\
\text { Gaps }\end{array}$ & $\begin{array}{c}\text { Sectoral } \\
\text { Gap Models }\end{array}$ \\
\hline County & Hourly & ResStock & Coms & tock & IGATE-E & $\begin{array}{c}\text { SERA } \\
\text { / EVI-Pro }\end{array}$ & $\begin{array}{l}\text { Core, Detailed } \\
\text { Sector Models }\end{array}$ \\
\hline
\end{tabular}




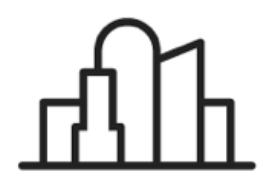

Housing stock characteristics database
Physics-based
High-performance

computing
EIA

NAHB

IECC

Census

EIA

NREL

NREL/Navigant
Res. Energy Consumption Survey (RECS) Homebuilder Surveys Historical Energy Codes
American Community Survey (ACS)

Electricity and fuel costs

OpenEl.org Utility Rate Database

Measure Cost Database

NREL
TMY3 weather data 


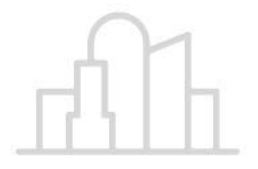

Housing stock characteristics

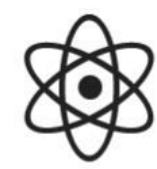

Physics-based computer modeling
High-performance

computing
U.S. DOE Tools

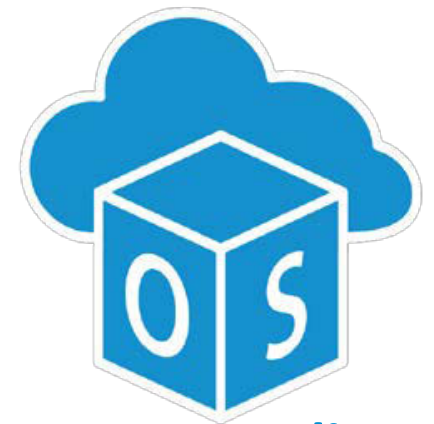

OpenStudio

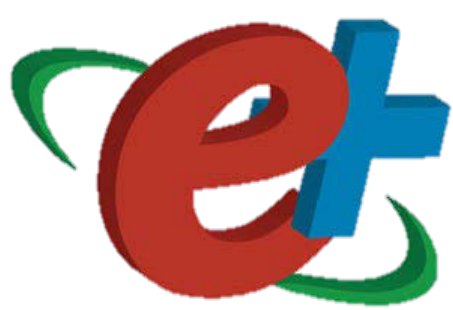

EnergyPlus
Detailed sub-hourly energy simulations

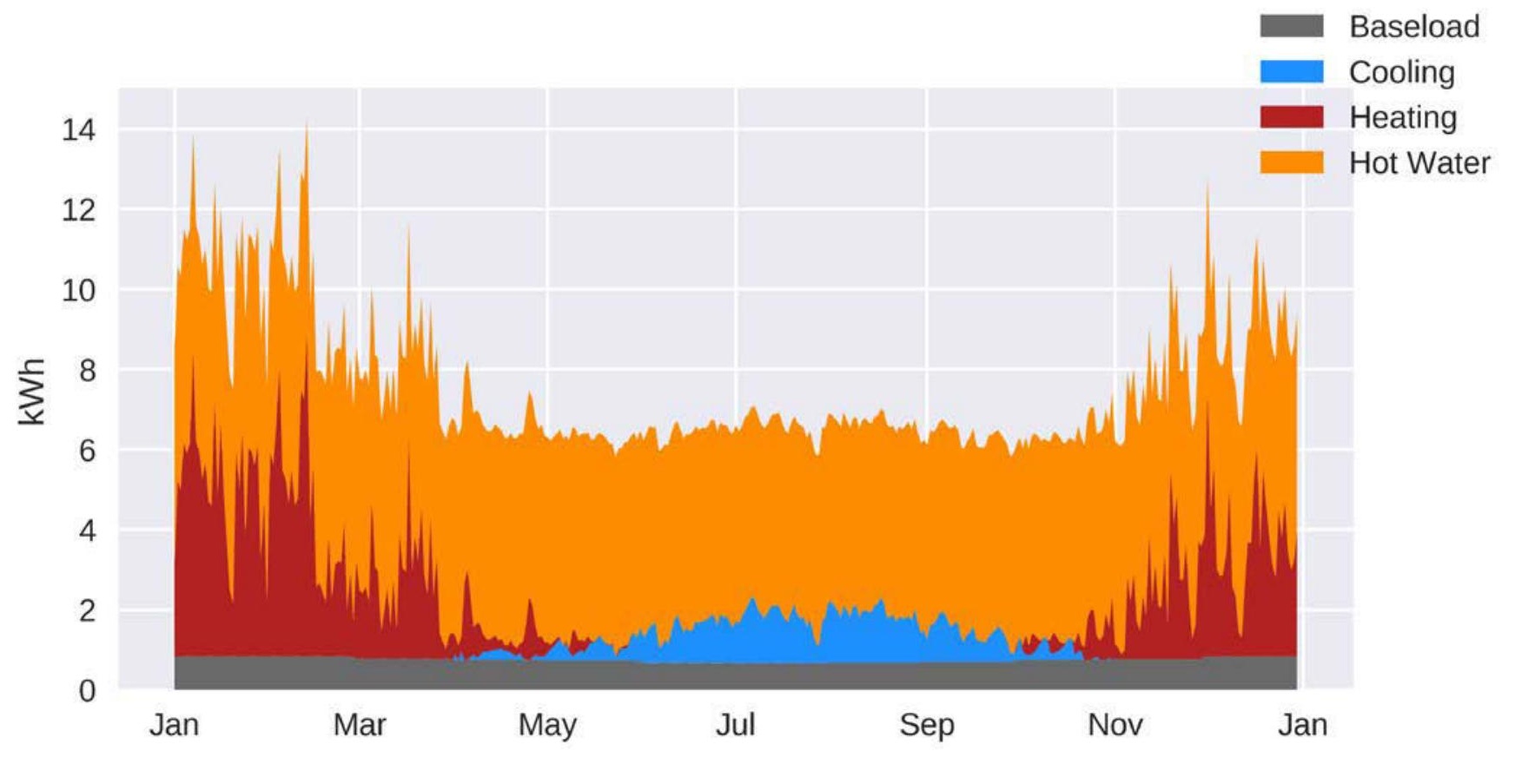




\section{Residential electricity load shapes by season}

- Interior equipment is the largest end use, comprising 33\% to $48 \%$ of electricity depending on census division

- Importance of space cooling, interior lighting, and space heating varies more by season and region
Weekday

Weekend
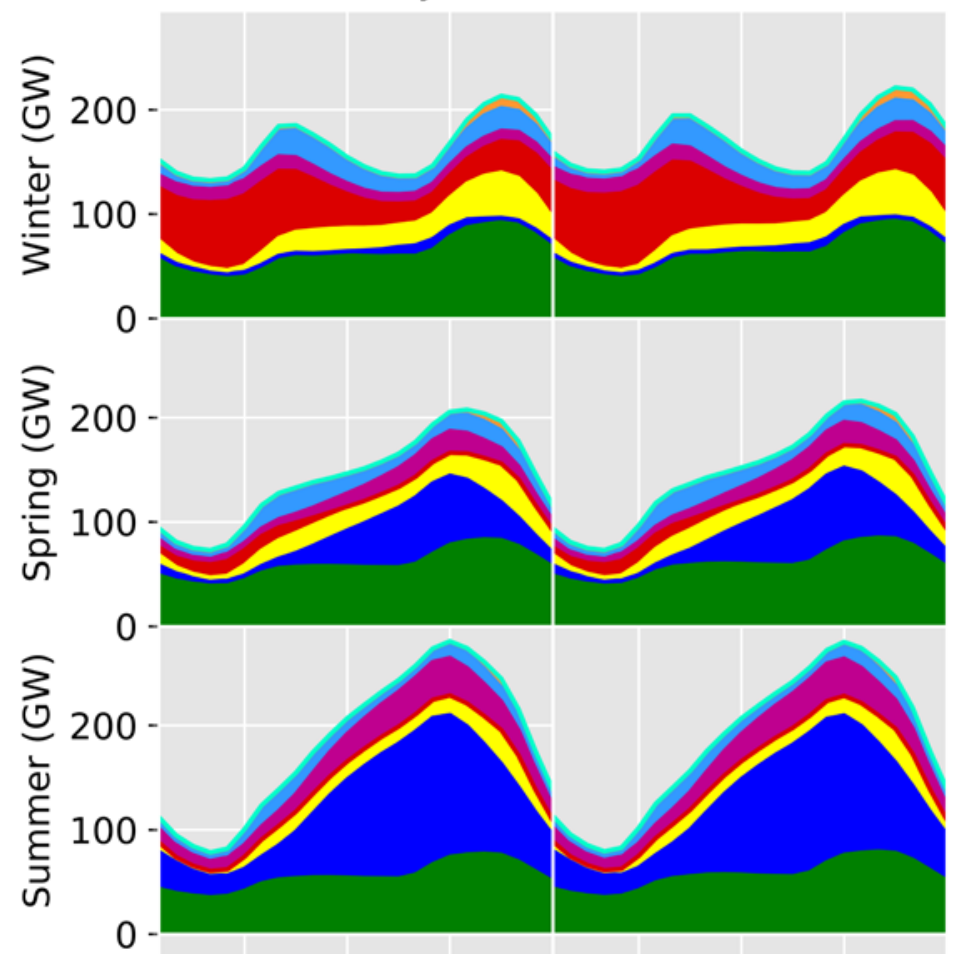

Heat Rejection Pumps

Exterior Lights Water Systems Fans

Space Heating

Interior Lights

Space Cooling

Interior Equipment

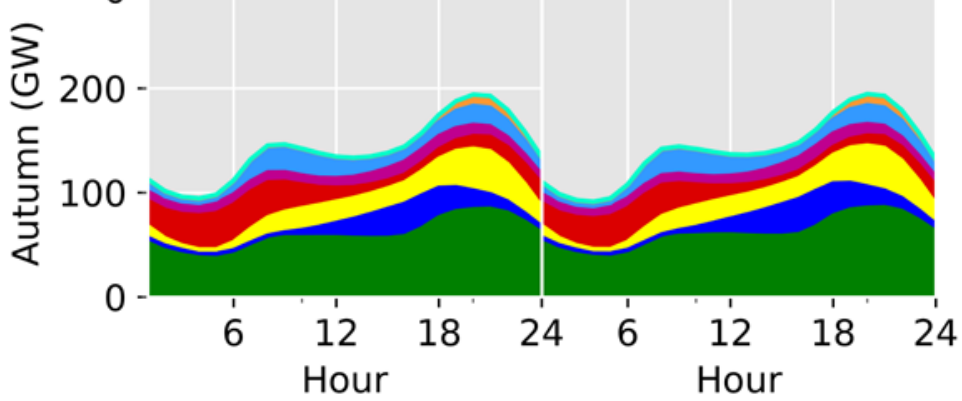



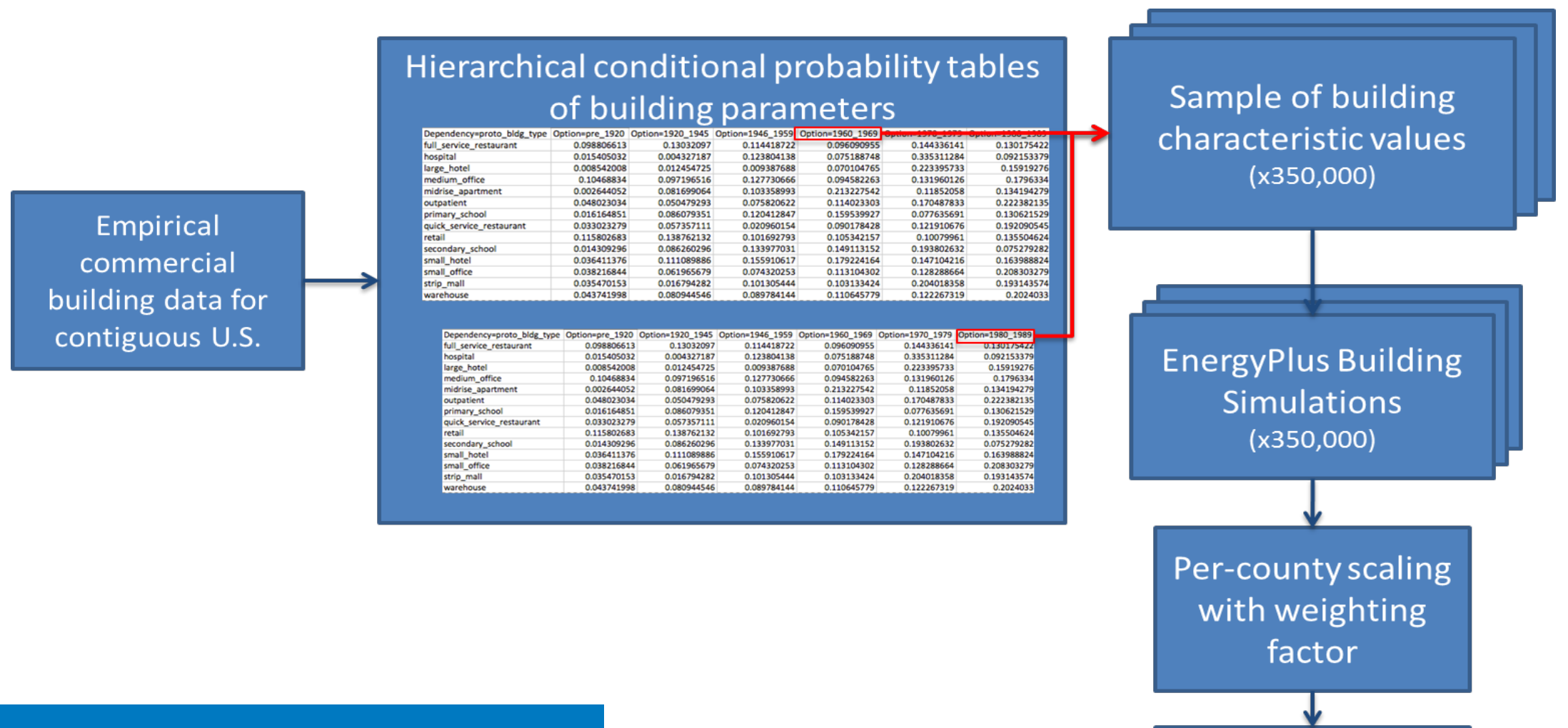

\section{ComStock:} commercial building modeling approach 


\section{Commercial building data relationships}

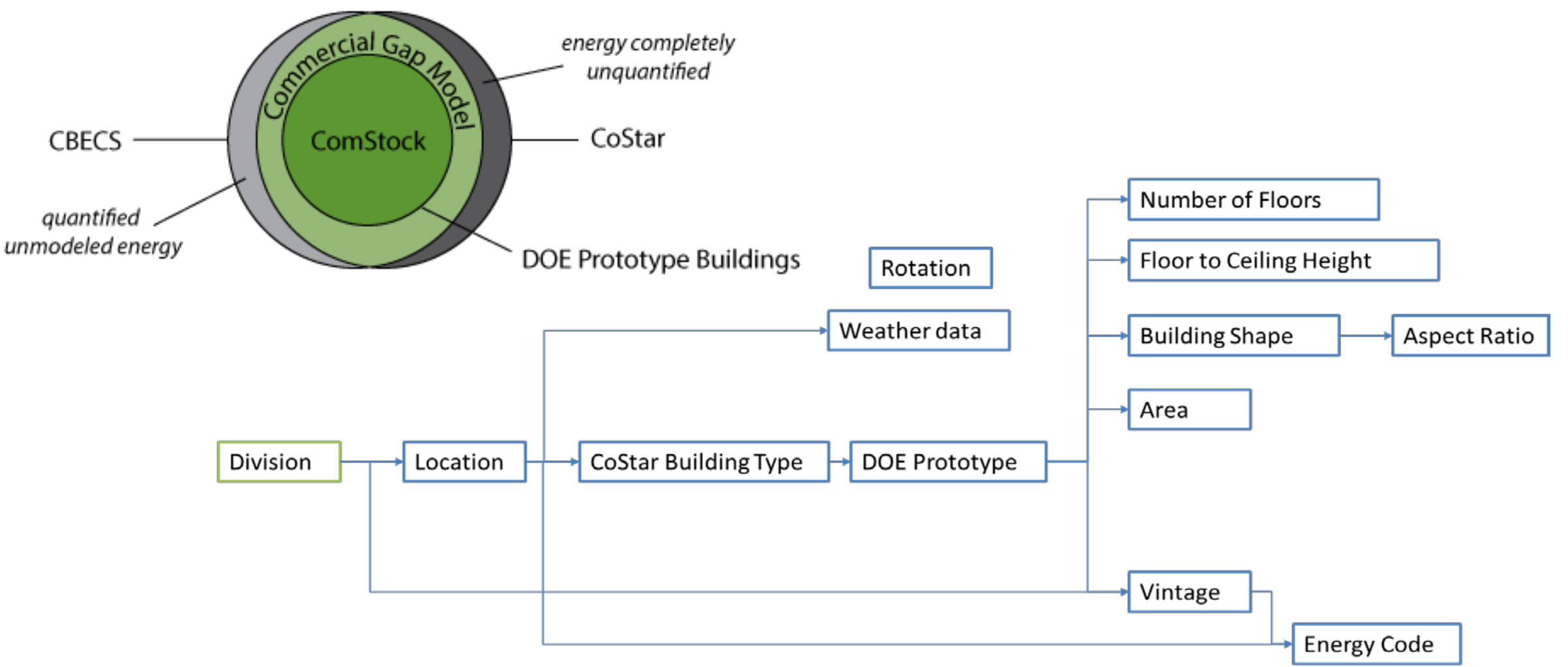




\section{Commercial electricity load shapes by season}

- More prominent role for lighting and fans, compared to residential buildings

- End use proportions vary by building type/subsector:

- Interior equipment is prominent in offices

- Interior lighting is prominent in retail buildings
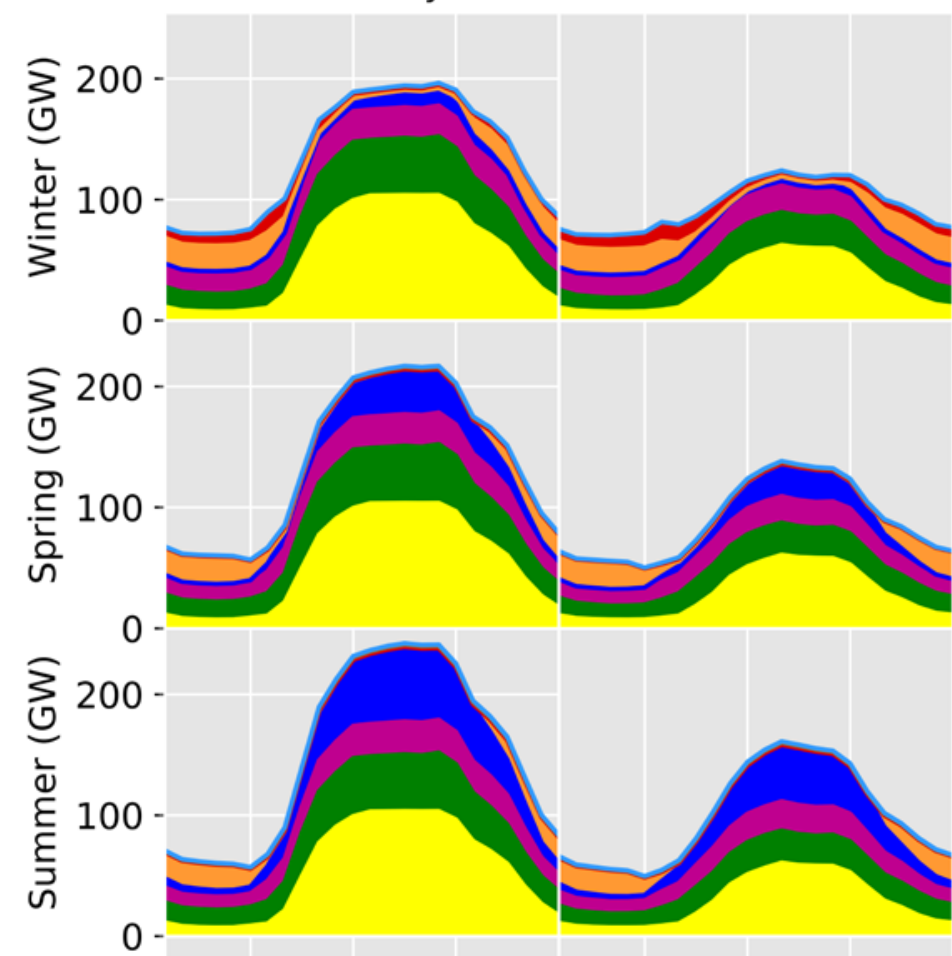

Systems Heat Rejection

Pumps

Space Heating

Exterior Lights

Space Cooling

Fans

Interior Equipment Interior Lights

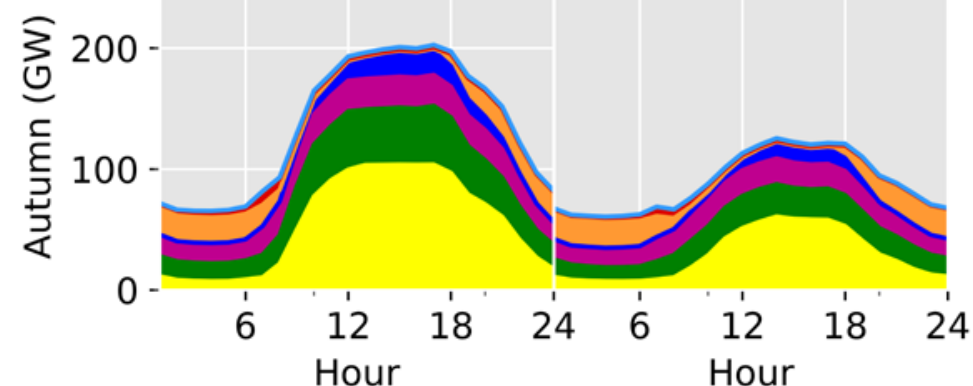


Industrial manufacturing modeling approach

\section{IGATE-E developed by ORNL and EPRI}

\begin{tabular}{|c|c|c|c|c|c|c|c|c|c|}
\hline & \multicolumn{5}{|c|}{ Methods } & \multicolumn{4}{|c|}{ Data Sources } \\
\hline Characteristics & 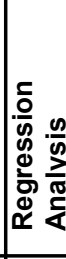 & 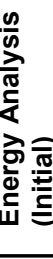 & 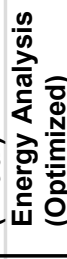 & 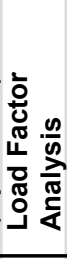 & 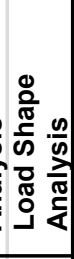 & \multirow[t]{2}{*}{ 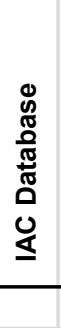 } & \multirow[t]{2}{*}{ 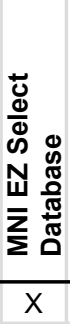 } & \multirow[t]{2}{*}{ 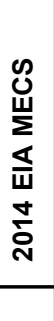 } & 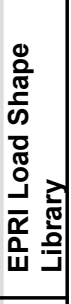 \\
\hline Location (State/County/Zip Code) & & $\mathrm{x}$ & & & & & & & \\
\hline Industry Code (NAICS/SIC) & $\mathrm{x}$ & $\mathrm{X}$ & & $\mathrm{x}$ & & $\mathrm{x}$ & $\mathrm{x}$ & & \\
\hline $\begin{array}{l}\text { Energy Consumption (kWh or } \\
\text { MMBtu/yr) }\end{array}$ & $\mathrm{x}$ & & & $\mathrm{x}$ & & $\mathrm{x}$ & & & \\
\hline Electricity Demand (kW/month) & & & & $\mathrm{x}$ & & $\mathrm{x}$ & & & \\
\hline Number of Employees & $\mathrm{x}$ & $\mathrm{x}$ & & & & $\mathrm{x}$ & $\mathrm{x}$ & & \\
\hline Industry Code (NAICS/SIC) & & & $x$ & & $x$ & & & $x$ & \\
\hline $\begin{array}{l}\text { Energy Consumption (kWh or } \\
\text { MMBtu/yr) }\end{array}$ & & & $\mathrm{x}$ & & & & & $\mathrm{x}$ & \\
\hline $\begin{array}{l}\text { End-Use Energy Consumption } \\
\text { (kWh/yr) }\end{array}$ & & & & & $\mathrm{x}$ & & & $\mathrm{x}$ & \\
\hline Load Shapes by End-Use & & & & & $\mathrm{x}$ & & & & $\mathrm{x}$ \\
\hline
\end{tabular}

Industrial Geospatial Analysis Tool for Energy Evaluation (IGATE-E)

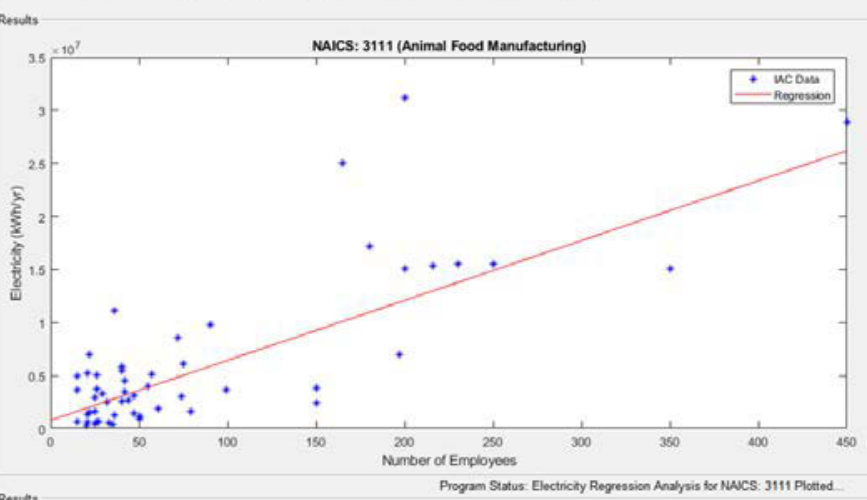

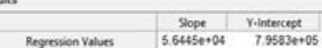

Pherabcuto Revits

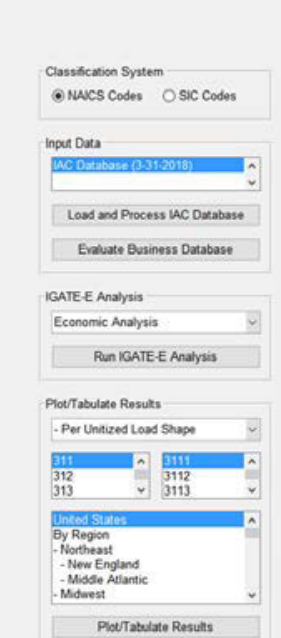

Industrial Geospatial Analysis Tool for Energy Evaluation (IGATE-E)

Resuins -

NAICS: 3111 (Animal Food Manutacturing)

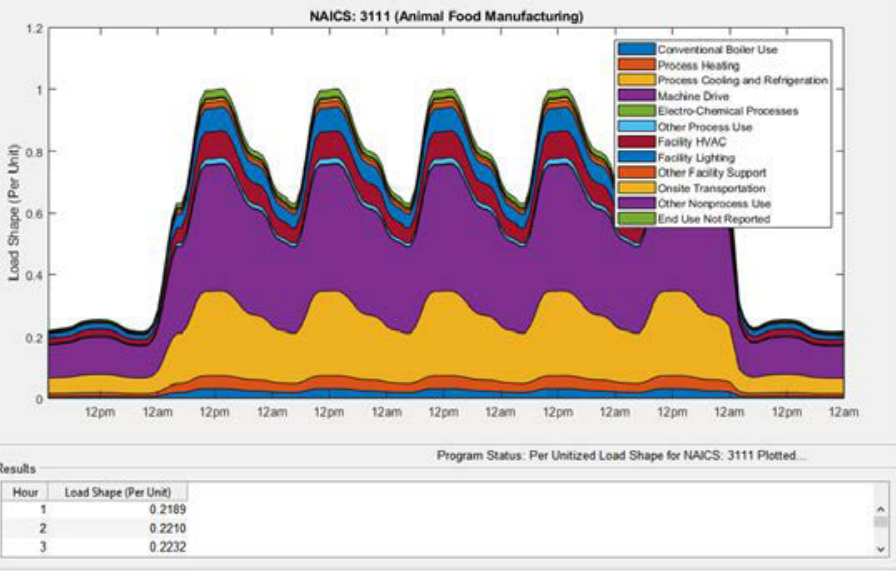




\section{Manufacturing electricity load shapes by season}

- Energy modeling is less developed in part because of industry / manufacturing heterogeneity

- IGATE-E/dsgrid models 86 different subsectors

- Electricity use is dominated by machine drive, process heating, and facility HVAC, with considerable subsector variation
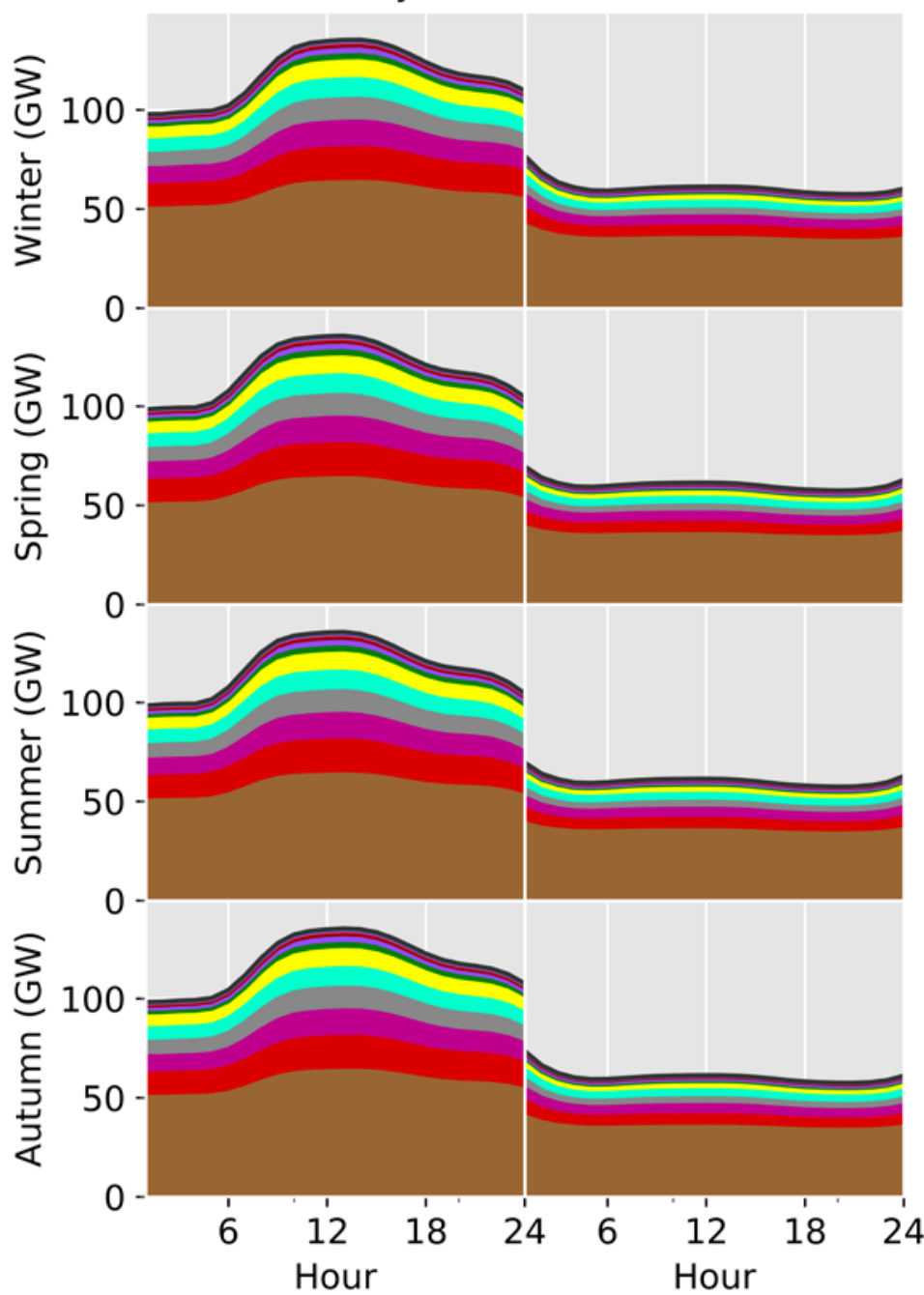

Other Nonprocess Use

Onsite

Transportation

End Use Not

Reported

Conventional

Boiler Use

Other Facility

Support

Other Process Use

Facility Lighting

Process Cooling

And Refrigeration

Electrochemical

Processes

Facility HVAC

Process Heating

Machine Drive 


\section{Transportation - electric vehicle modeling approach}

- Overall electrification ambition

- LDV/MDV/HDV role

Scenario Definition

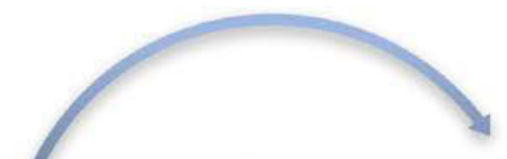

Vehicle Adoption

- Based on cost, performance, and projected improvement
- Based on spatial distribution of early adopters

Regionalization of Vehicle Deployment

\section{Real-World} Hourly Power Profiles

- Based on travel surveys, vehicle characteristics, and charging assumptions 


\section{Transportation - charging profiles from EVI-Pro}

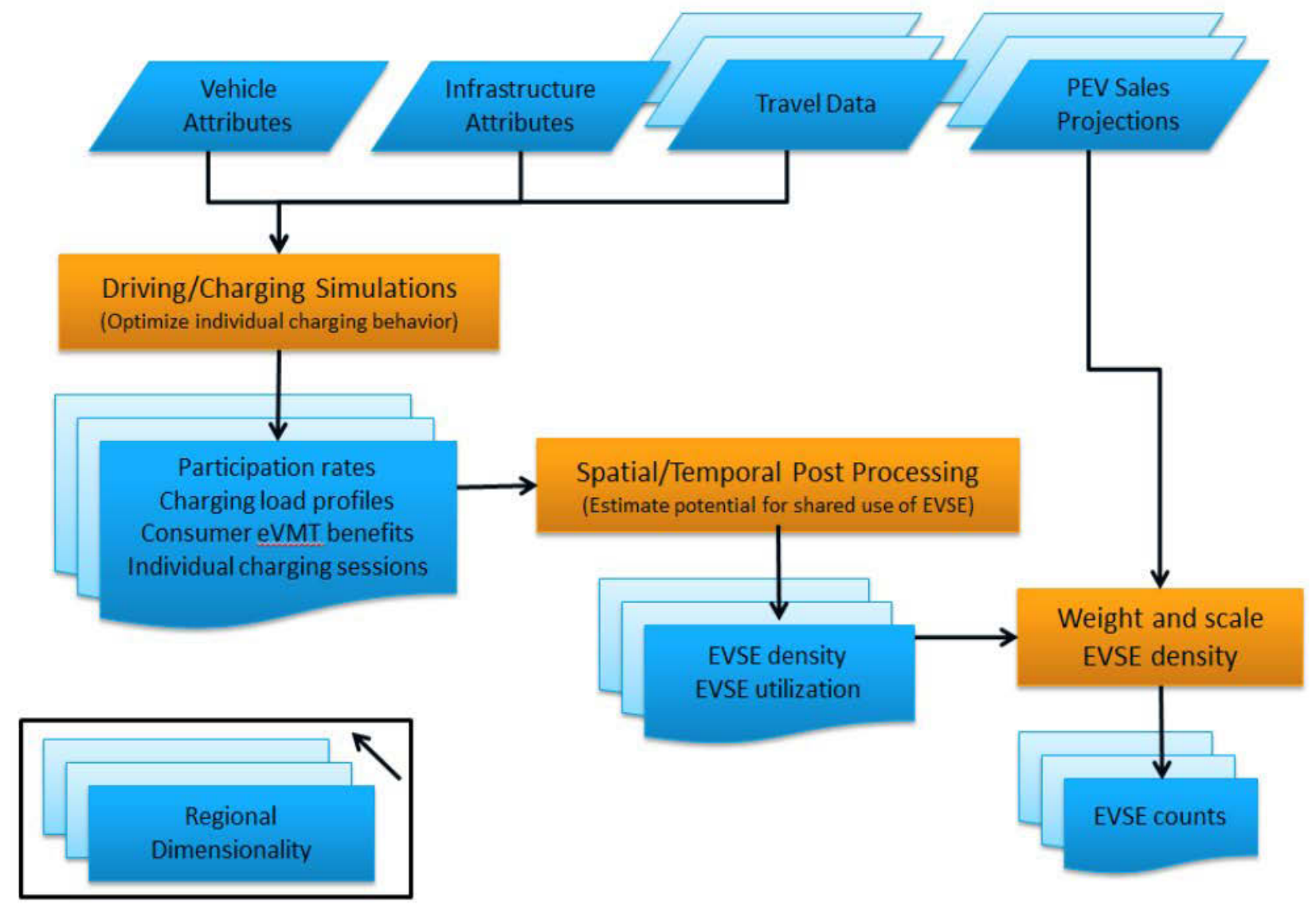




\section{Historical Calibration Data Sources}

Planning

Region

Hourly

ISO / FERC 714 System Generation Data

Utility

by State

Utility

by State
Annual

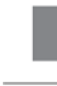

Annual

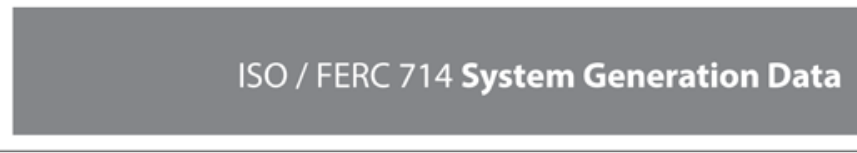

EIA 861 Operational Data on System Losses

\begin{tabular}{|c|c|c|}
\hline Residential & Commercial & Industrial \\
\hline nents & & \\
\hline $\mathrm{CHP}$ & $\mathrm{CHP}$ & $\mathrm{CHP}$ \\
\hline DPV & DPV & DPV \\
\hline
\end{tabular}

Centralized Supply

\section{T \& D Losses}

\begin{tabular}{c|c} 
Transport & $\begin{array}{l}\text { EIA } 861 \text { Retail Sales } \\
\text { \& Operational Data }\end{array}$
\end{tabular}

Site-Level Supply

\section{dsgrid Model Components}

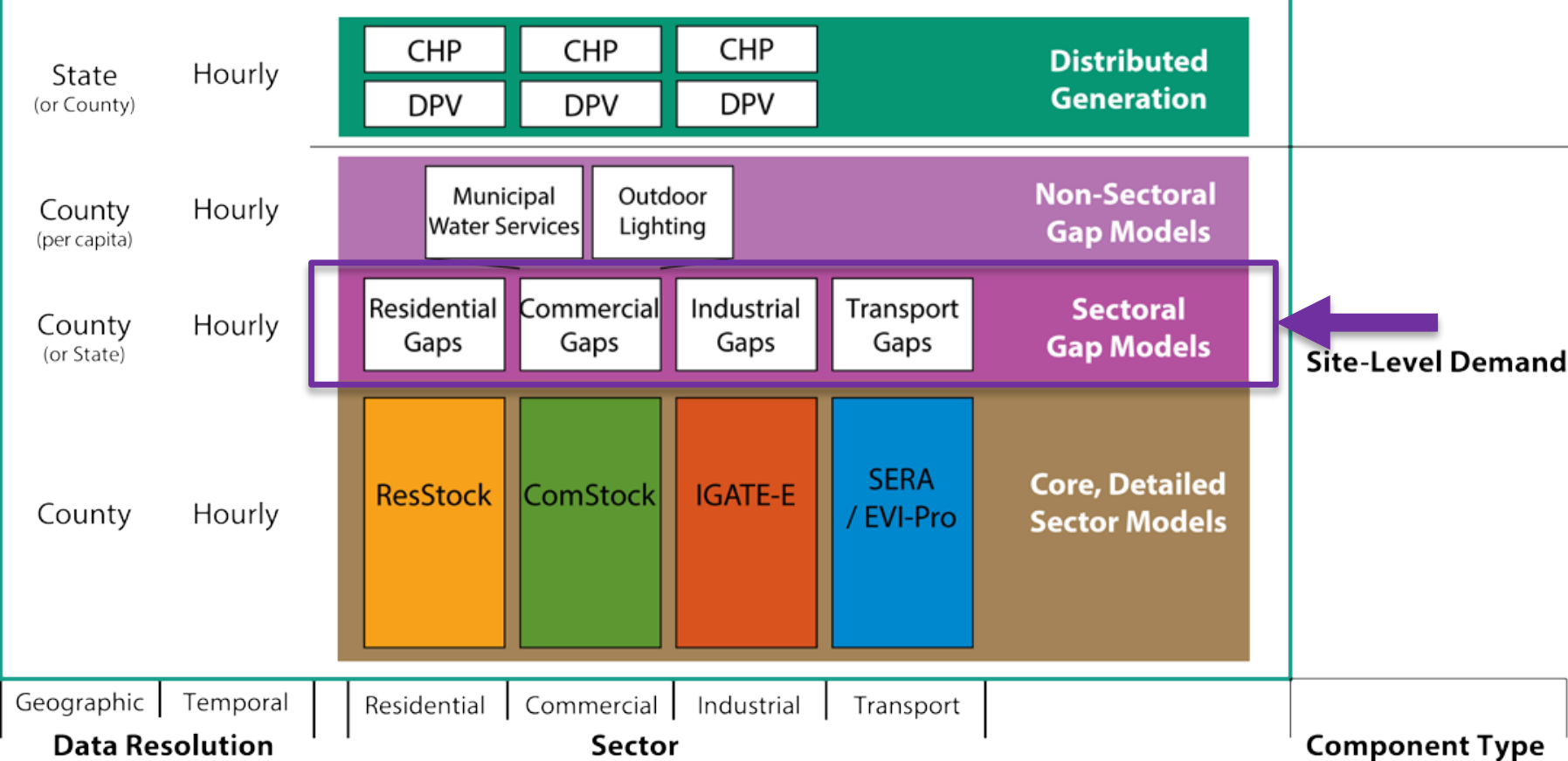


Transportation gap model of electricity used by passenger trains

\begin{tabular}{lrr}
\hline Census Division & Total GWh) & \multicolumn{1}{c}{ Total (\%) } \\
\hline Mid Atlantic & 3,304 & 51.5 \\
South Atlantic & 793 & 12.4 \\
New England & 709 & 11.0 \\
Pacific & 706 & 11.0 \\
East North Central & 623 & 9.7 \\
Mountain & 124 & 1.9 \\
West South Central & 114 & 1.8 \\
West North Central & 43 & 0.7 \\
East South Central & 2 & 0.0 \\
\hline Total & 6,417 & 100.0 \\
\hline
\end{tabular}

Electric vehicles not modeled in the 2012 data set because deployment at that time was small and regionally concentrated
Seasonal, weekday/weekend load shapes for CONUS
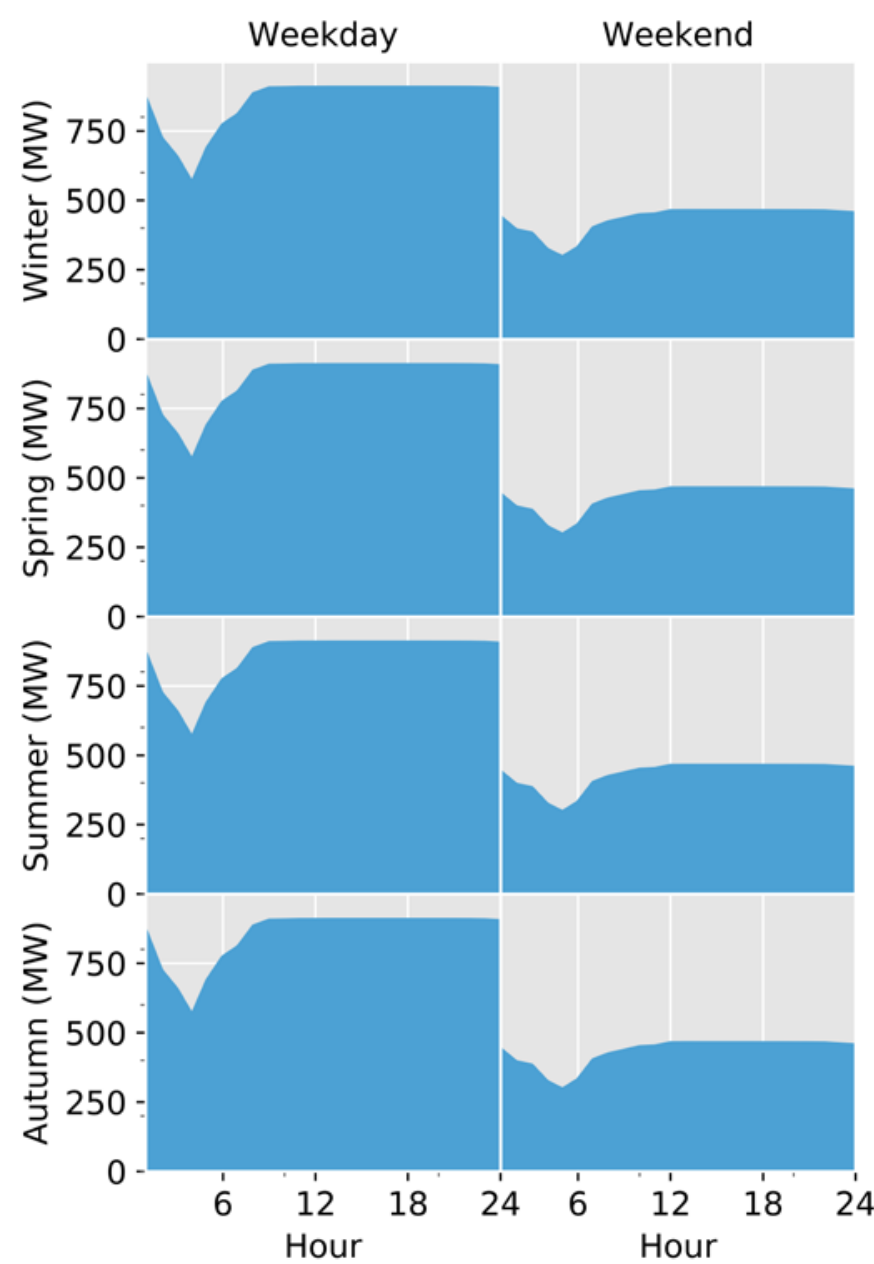


\section{dsgrid models about $80 \%$ of 2012 U.S. electricity use in detail}

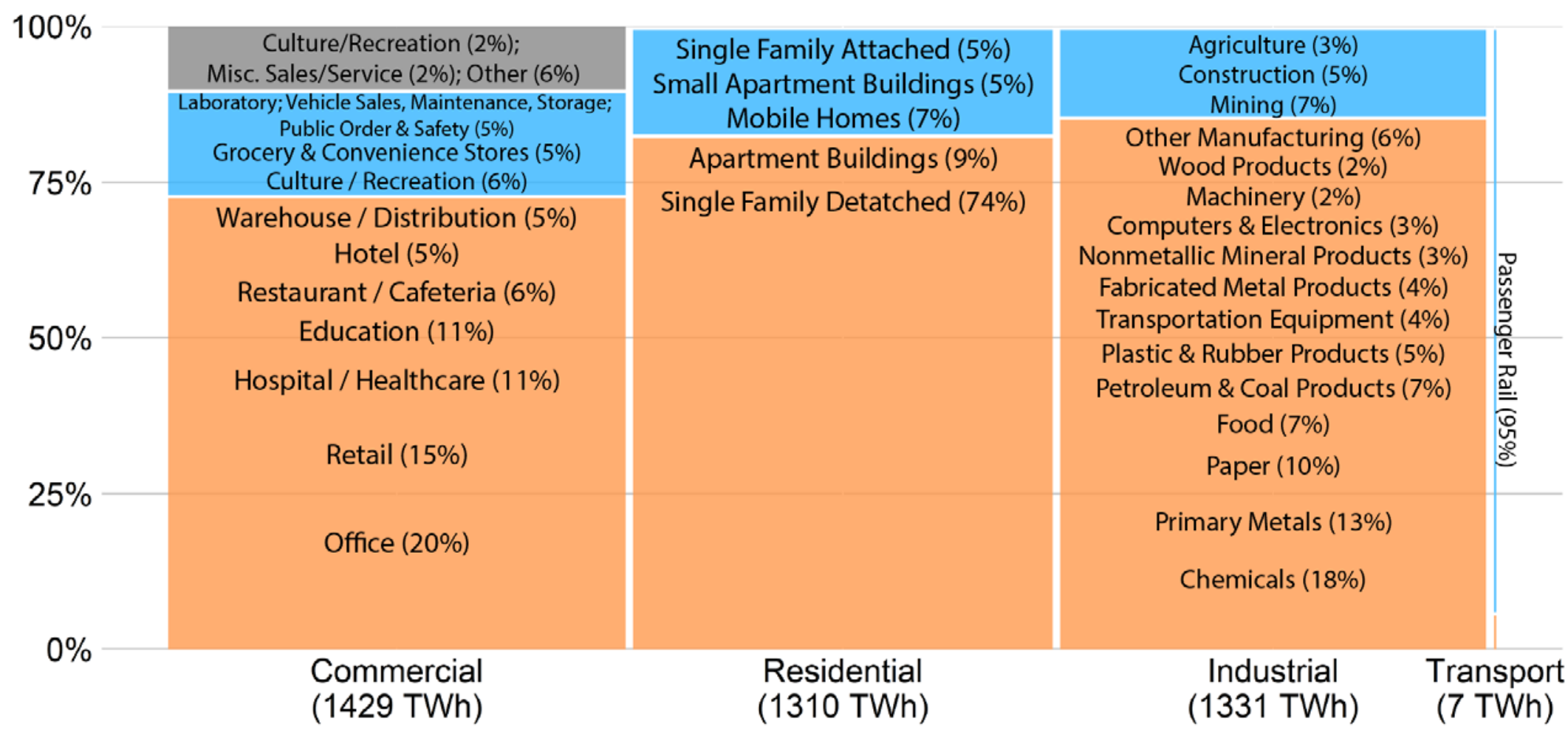

Subsectors in each category are listed with the percentage of electricity they use relative to the sector's electricity use.

Detailed Gap Unmodeled 


\section{dsgrid models about $76 \%$ of 2012 U.S. site energy use in detail}

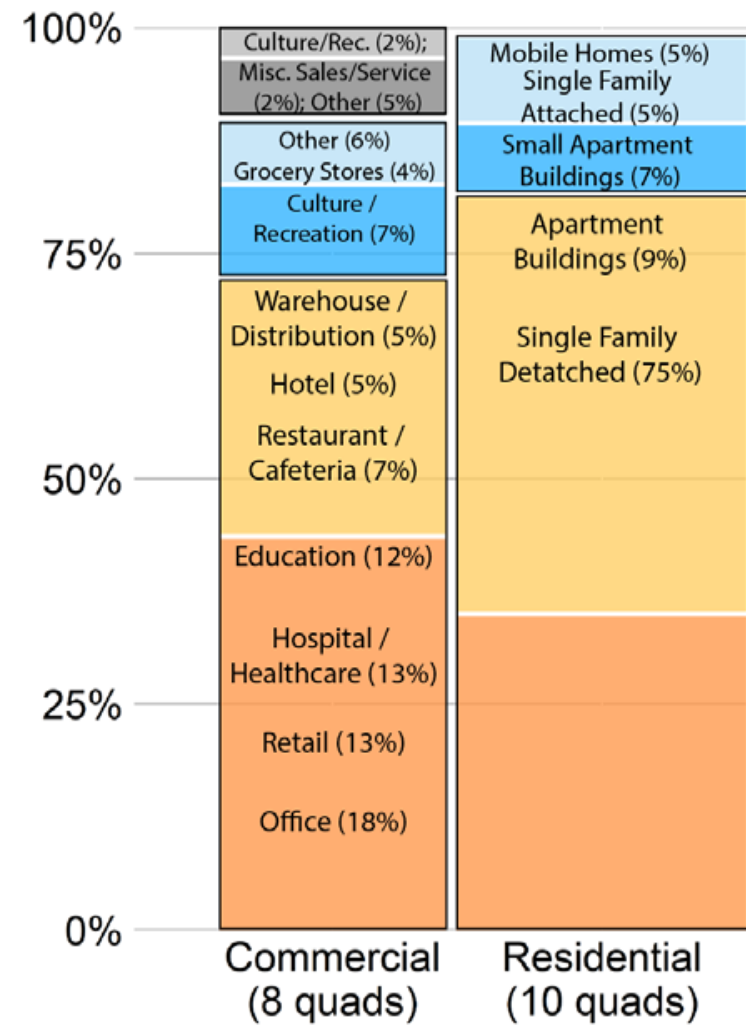

\begin{tabular}{|c|}
\hline Agriculture (5\%) \\
Construction (10\%) \\
Mining (17\%) \\
\hline Other Manufacturing (7\%) \\
Plastic \& Rubber Products (2\%) \\
Transportation Equipment (3\%) \\
Fabricated Metal Products (3\%) \\
Paper (5\%) \\
Nonmetallic Mineral \\
Products (6\%) \\
Food (6\%) \\
Primary Metals (9\%) \\
Petroleum \& Coal \\
Products (10\%) \\
Chemicals (17\%) \\
\hline Industrial \\
(15 quads)
\end{tabular}

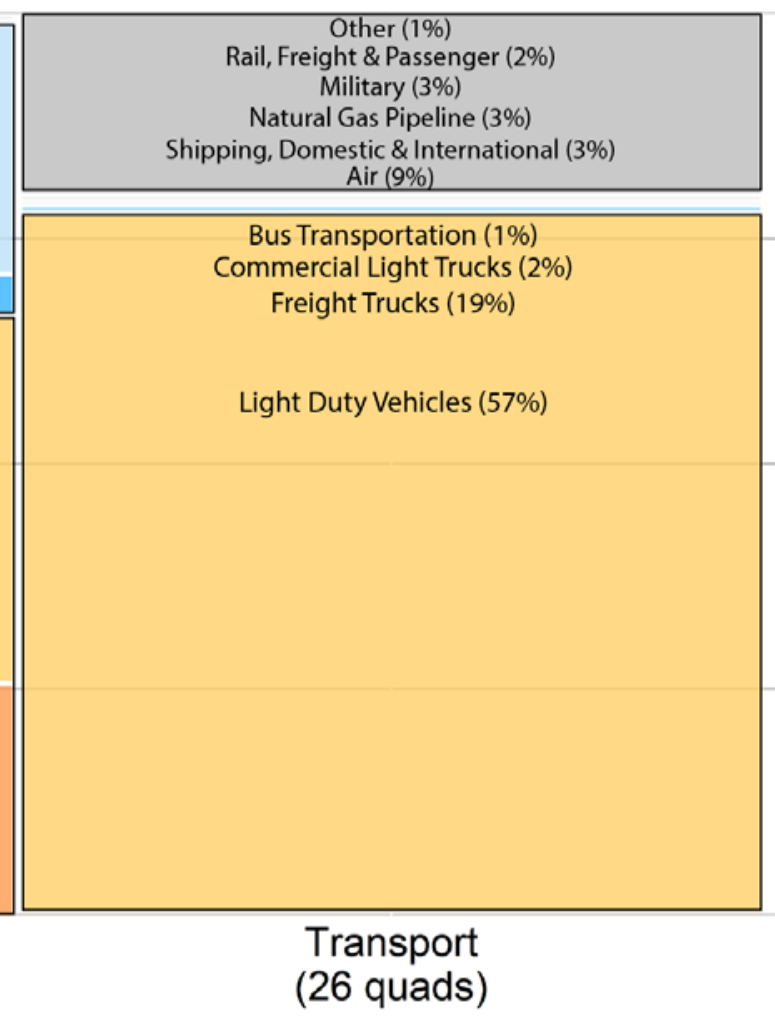

Subsectors in each category are listed with the percentage of all energy they use relative to the sector's overall energy use. 


\section{Historical Calibration Data Sources}

Planning

Region

Hourly

ISO / FERC 714 System Generation Data

Utility

by State

Utility

by State
Annual

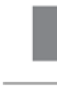

Annual

\section{dsgrid Model Components}

\begin{tabular}{|c|c|c|c|c|c|c|c|}
\hline $\begin{array}{l}\text { State } \\
\text { (or County) }\end{array}$ & Hourly & $\frac{\text { CHP }}{\text { DPV }}$ & $\frac{\mathrm{CH}}{\mathrm{DF}}$ & & $\frac{\text { CHP }}{\text { DPV }}$ & & $\begin{array}{l}\text { Distributed } \\
\text { Generation }\end{array}$ \\
\hline $\begin{array}{l}\text { County } \\
\text { (per capita) }\end{array}$ & Hourly & $\begin{array}{r}\text { Muni } \\
\text { Water S }\end{array}$ & $\begin{array}{l}\text { ipal } \\
\text { rvices }\end{array}$ & $\begin{array}{l}\text { Outd } \\
\text { Light }\end{array}$ & & & $\begin{array}{c}\text { Non-Sectoral } \\
\text { Gap Models }\end{array}$ \\
\hline $\begin{array}{l}\text { County } \\
\text { (or State) }\end{array}$ & Hourly & \begin{tabular}{|c|} 
Residential \\
Gaps
\end{tabular} & $\begin{array}{r}\text { Comm } \\
\mathrm{Ga}\end{array}$ & ercial & $\begin{array}{l}\text { Industrial } \\
\text { Gaps }\end{array}$ & $\begin{array}{l}\text { Transport } \\
\text { Gaps }\end{array}$ & $\begin{array}{c}\text { Sectoral } \\
\text { Gap Models }\end{array}$ \\
\hline County & Hourly & ResStock & Coms & tock & IGATE-E & $\begin{array}{c}\text { SERA } \\
\text { / EVI-Pro }\end{array}$ & $\begin{array}{l}\text { Core, Detailed } \\
\text { Sector Models }\end{array}$ \\
\hline
\end{tabular}

EIA 861 Operational Data on System Losses

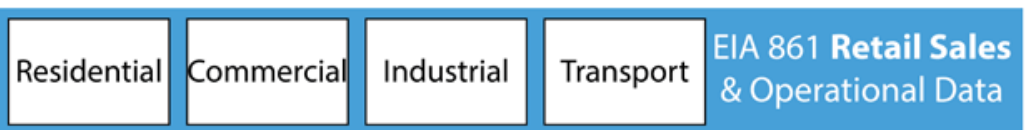

Centralized Supply

T \& D Losses

Site-Level Supply

Site-Level Demand 


\section{Municipal services not captured in the sector models}

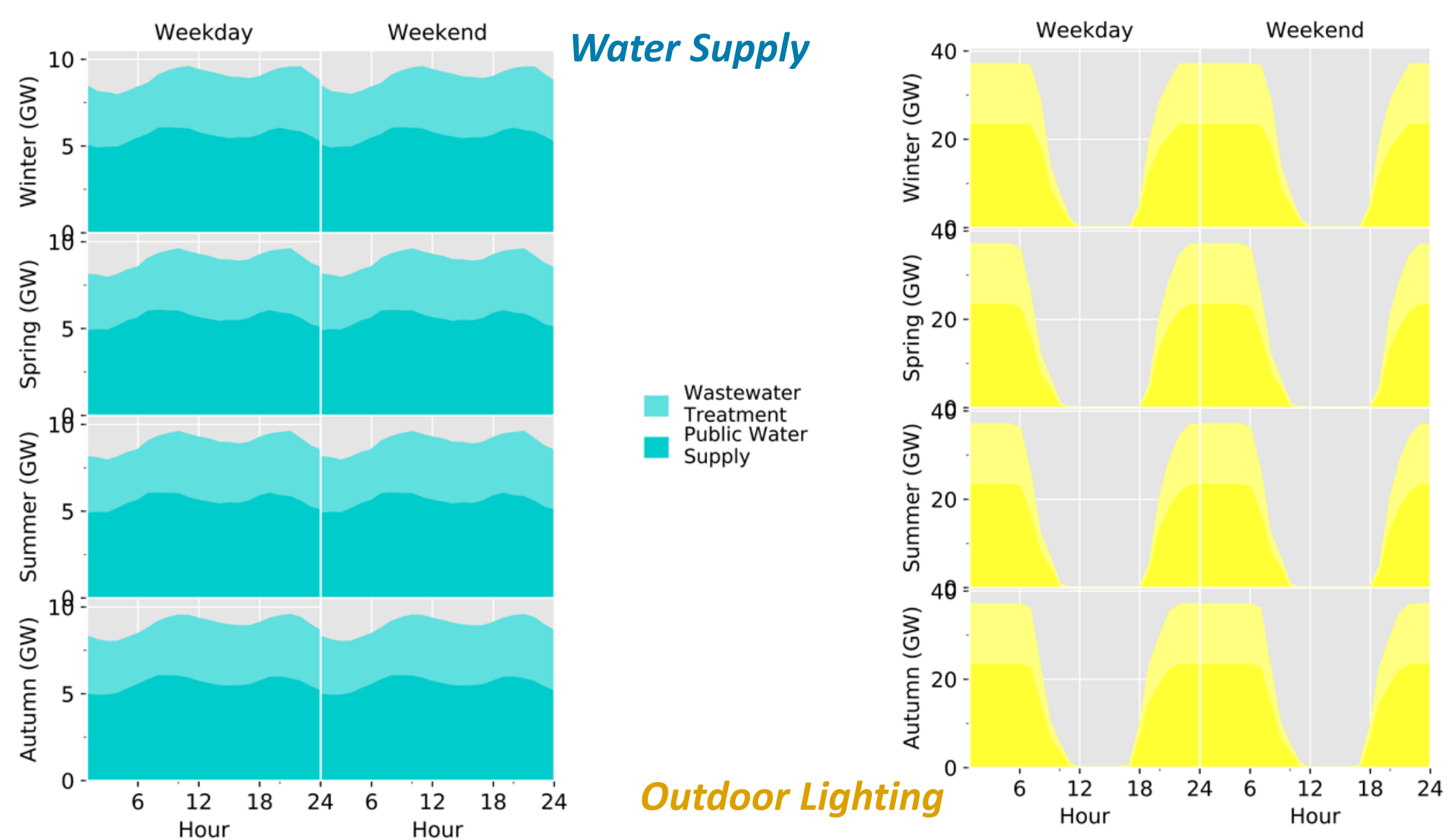




\section{Validation against historical data requires distributed generation estimates}

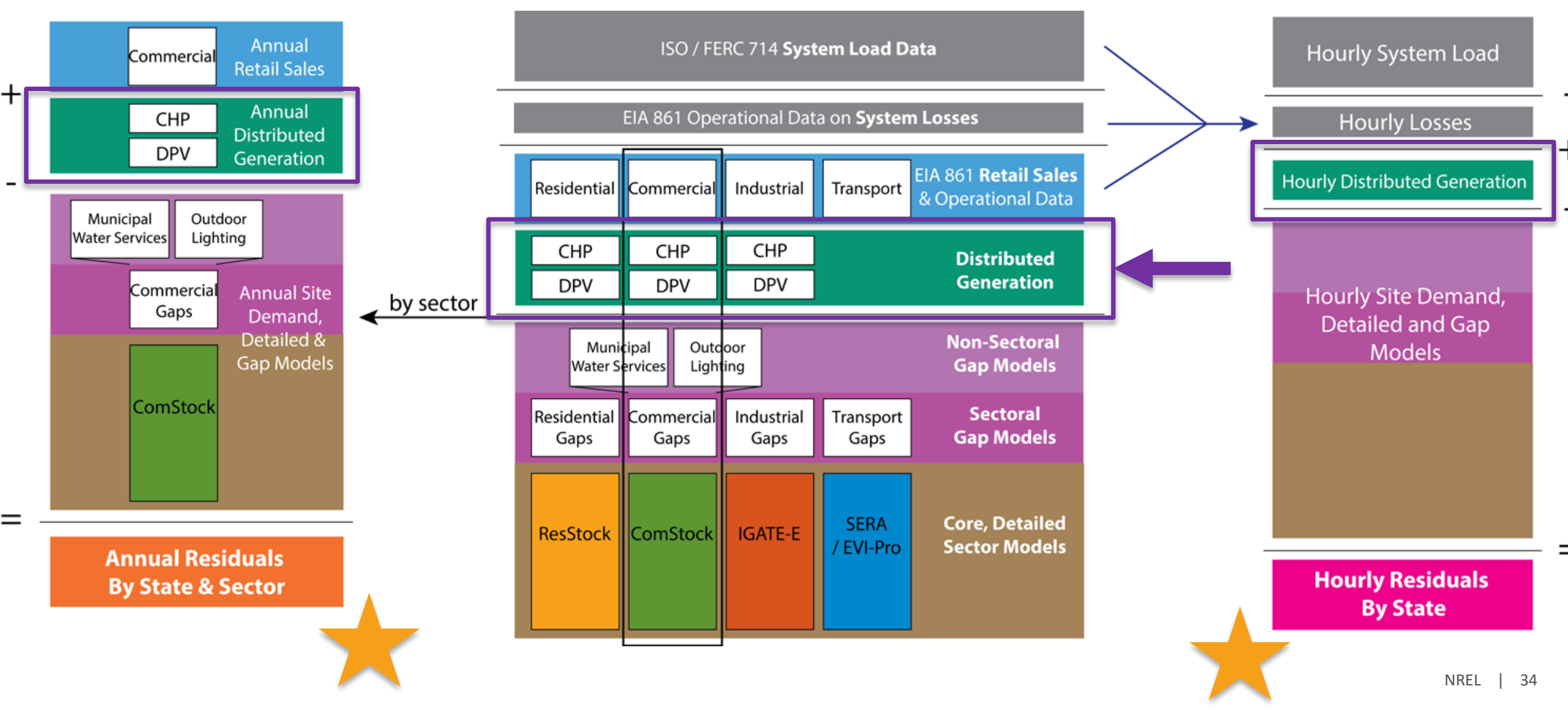




\section{Distributed solar and combined heat and power}

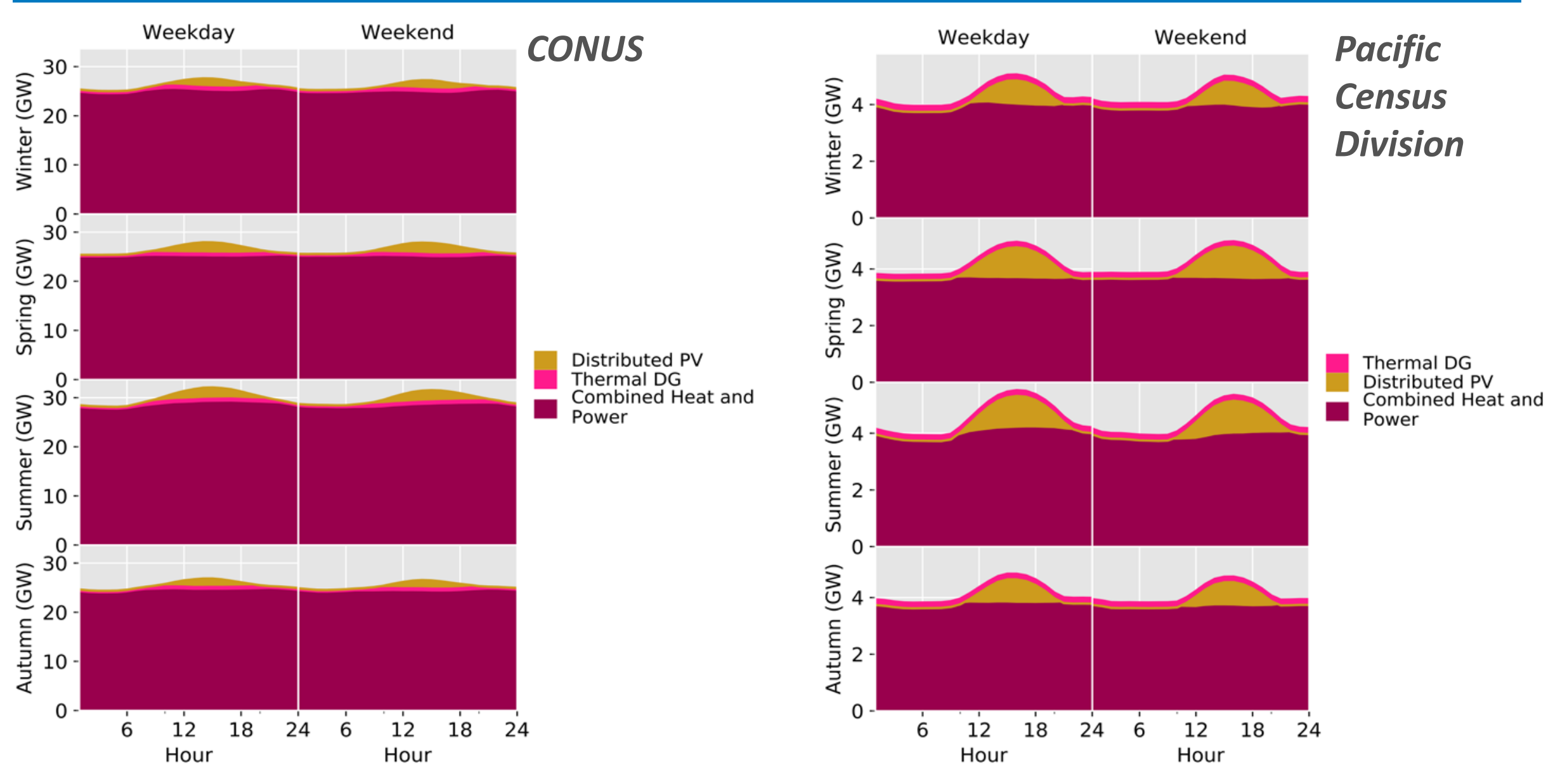




\section{Hourly data roughly validates, but also reveals need for additional calibration}

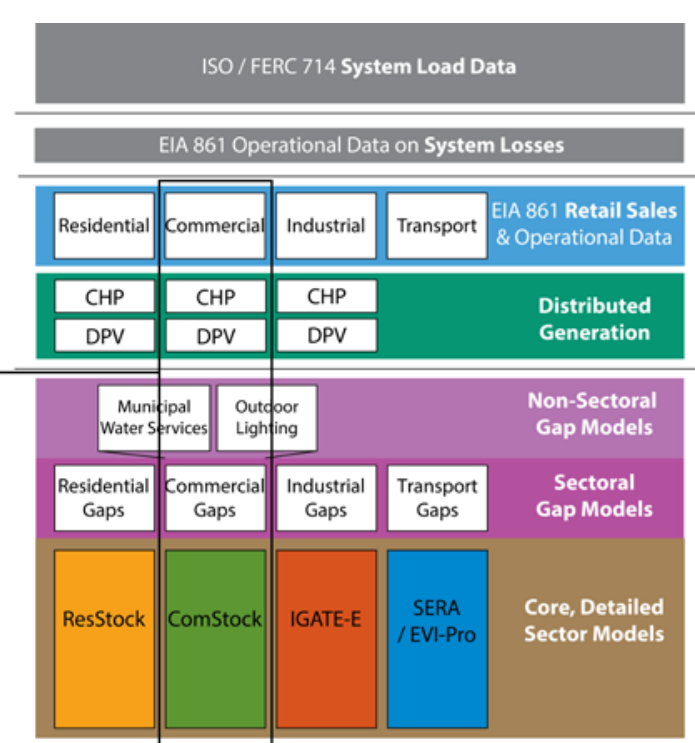

Hourly System Load

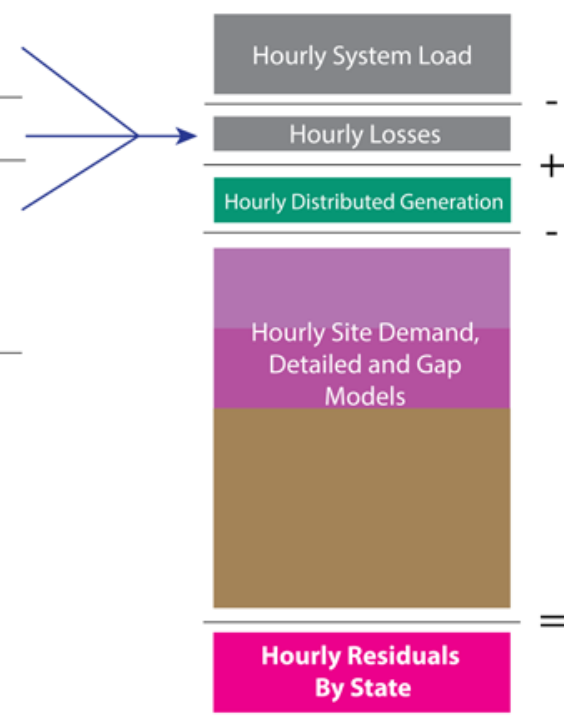

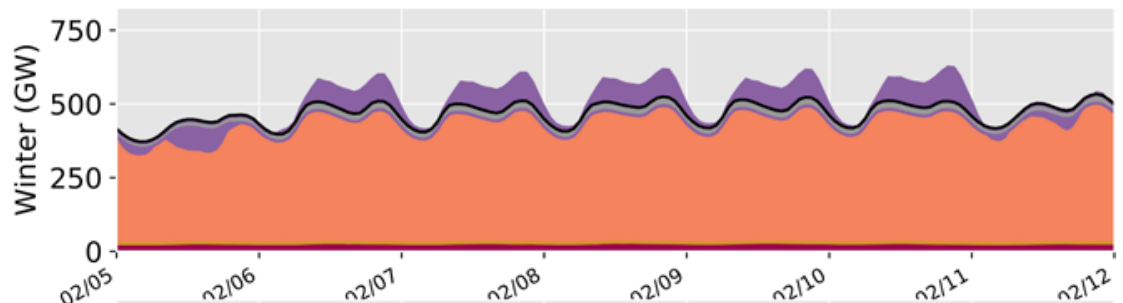
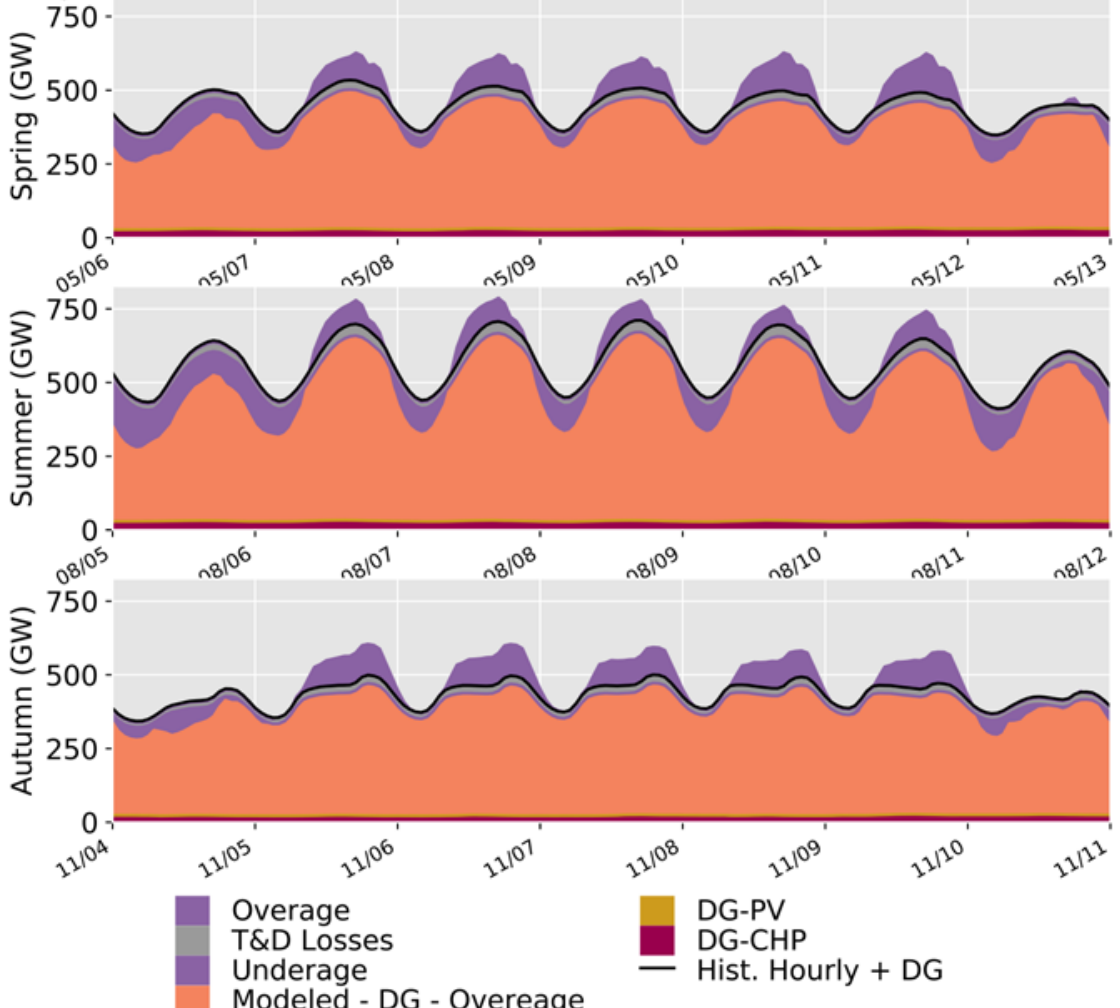


\section{Annual sector-level residuals reveal a similar story}
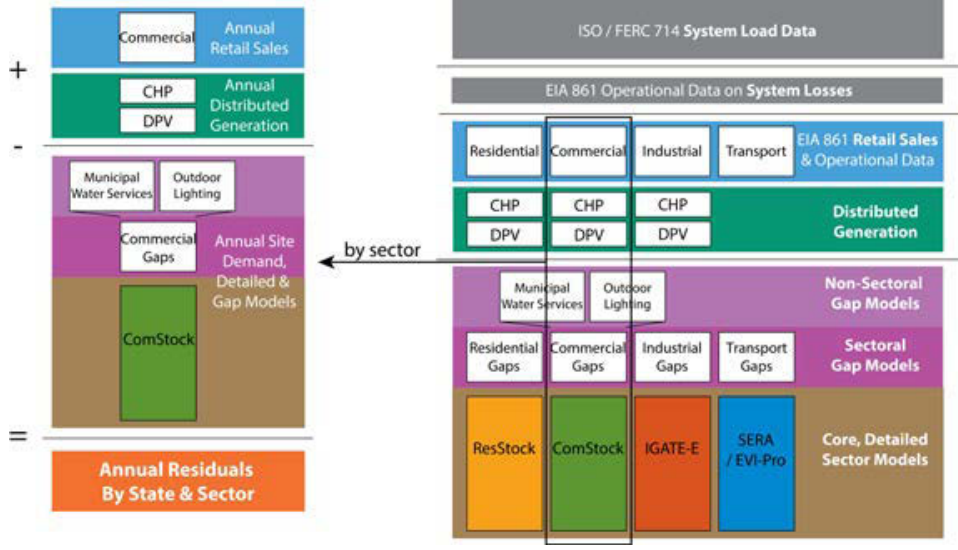

\begin{tabular}{|c|c|c|c|c|c|c|}
\hline $\begin{array}{l}\text { Component } \\
\text { Type }\end{array}$ & $\begin{array}{l}\text { Component } \\
\text { Name }\end{array}$ & $\begin{array}{l}\text { Residential } \\
\text { (TWh) }\end{array}$ & $\begin{array}{c}\text { Commercial } \\
\text { (TWh) }\end{array}$ & $\begin{array}{c}\text { Industrial } \\
\text { (TWh) }\end{array}$ & $\begin{array}{c}\text { Transport } \\
\text { (TWh) }\end{array}$ & Total (TWh) \\
\hline Top-down & Hourly load & & & & & 3,910 \\
\hline Derived & T\&D losses & & & & & 199 \\
\hline Top-down & Annual energy & 1,370 & 1,350 & 981 & 7 & 3,708 \\
\hline dsgrid & Distributed generation & 3 & 31 & 204 & - & 237 \\
\hline dsgrid-core & Gap models & 218 & 454 & 184 & 6 & 862 \\
\hline dsgrid-core & Detailed sector models & 1,169 & 1,107 & 893 & - & 3,170 \\
\hline Derived & Total site energya & 1,372 & 1,381 & 1,184 & 7 & 3,945 \\
\hline Derived & Annual sector residuals ${ }^{b}$ & -15 & -180 & 107 & 1 & -87 \\
\hline Derived & Hourly residuals & & & & & -126 \\
\hline
\end{tabular}

a Total site energy is the top-down annual energy plus distributed generation. This is all the load we are expecting to model with the bottom-up detailed sector and gap models.

b The sector level residuals are equal to the total site energy minus the gap and detailed sector model components. 


\section{Summary model fit statistics}
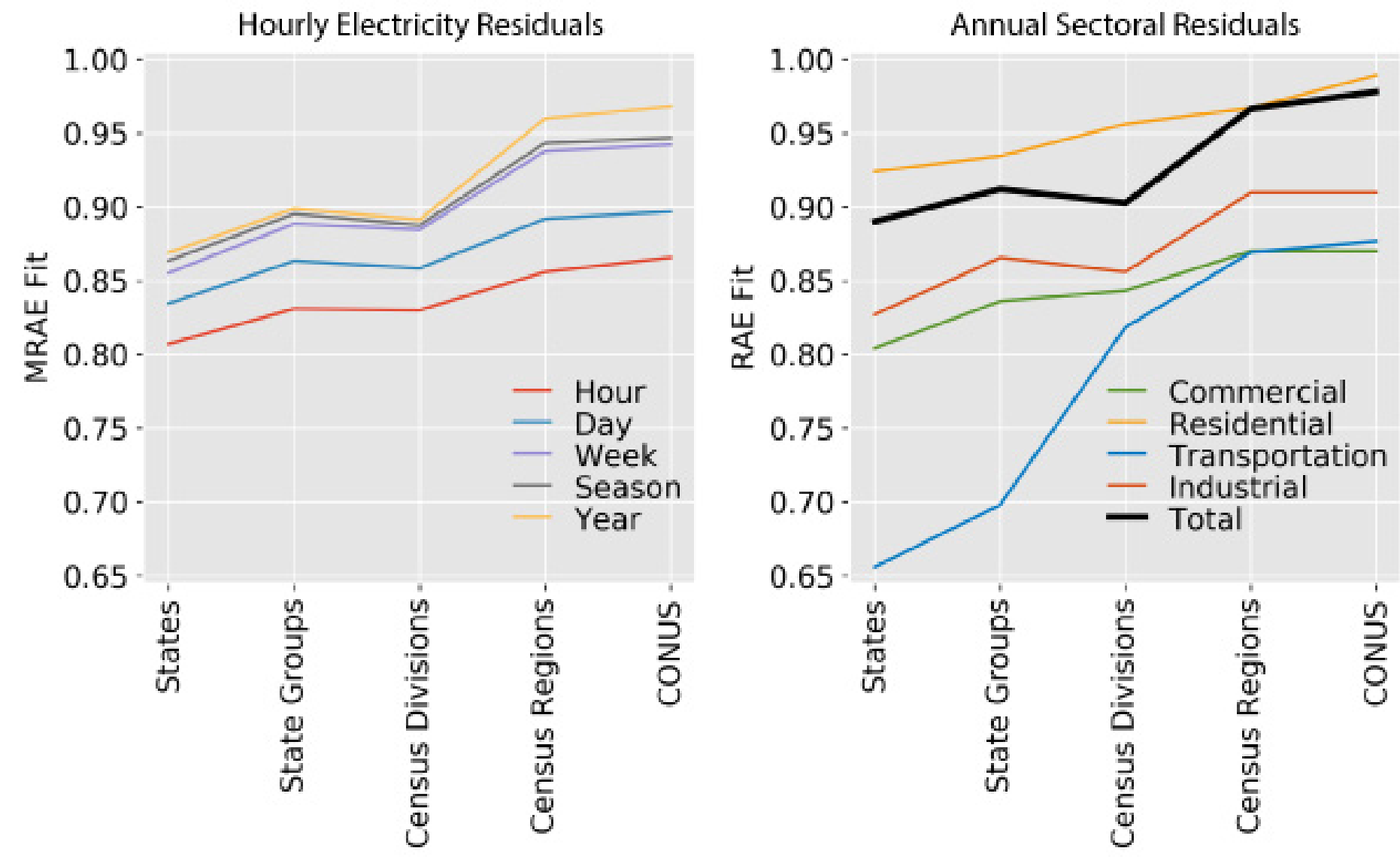


\section{Next steps}

Ongoing and planned work to:

- Create future load data sets (electrification, load modernization)

- Estimate flexibility potential

- Model demand response resource/programs in grid models

Electrification Futures Study plan to develop future load scenario snapshots for production cost modeling

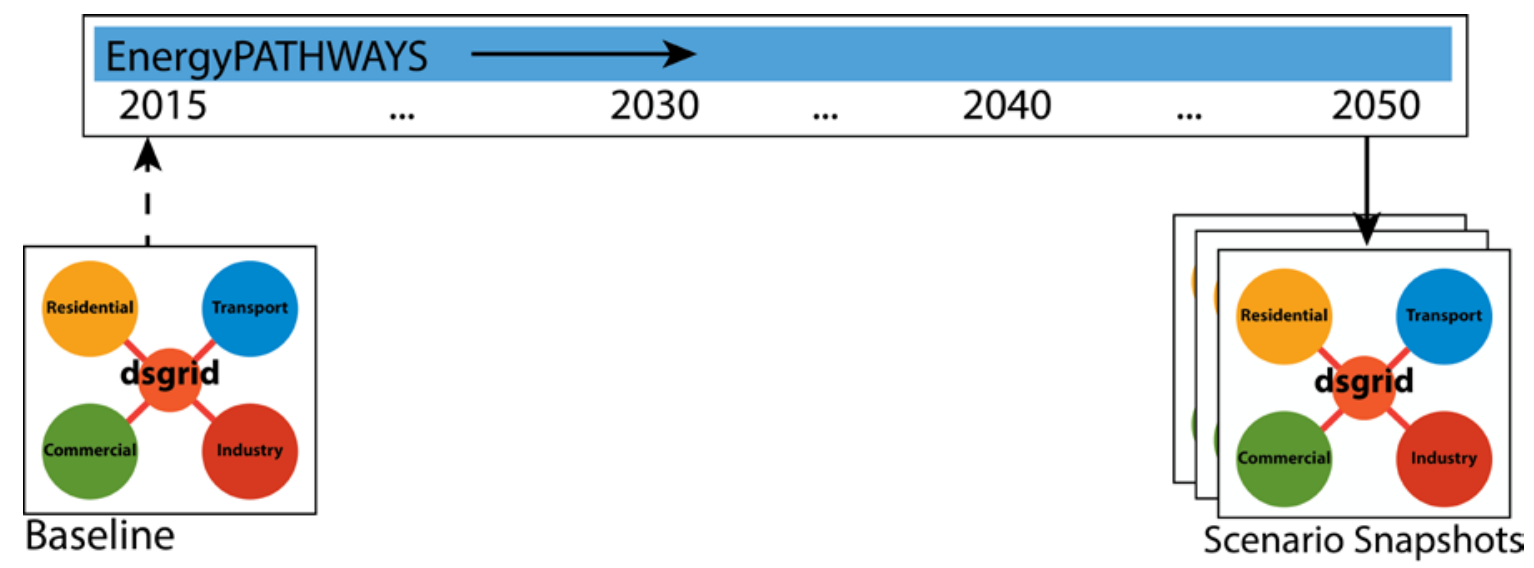




\section{Modeling Leads}

\section{dsgrid team}

Elaine Hale - Coordination Lead
$\begin{aligned} & \text { Ph.D. Chemical Engineering } \\ & \text { University of Texas, Austin } \\ & \text { elaine.hale@nrel.gov }\end{aligned}$

Co-authors: Brennan Borlaug, Craig Christensen, Amanda Farthing, Dylan Hettinger, Andrew Parker, Joseph Robertson, Michael Rossol, Gord Stephen, Eric Wood (NREL), Baskar Vairamohan (EPRI) 


\section{References}

Alkadi, Nasr, Michael Starke, Ookie Ma, Sachin Nimbalkar, Daryl Cox, Kevin Dowling, Brendon Johnson, and Saqib Khan. 2013. "Industrial Geospatial Analysis Tool for Energy Evaluation--IGATE-E." In Proceedings of the Thirty-Fifth Industrial Energy Technology Conference. New Orleans, LA. https://oaktrust.library.tamu.edu/bitstream/handle/1969.1/149152/ESL-IE-13-0513.pdf?sequence $=1$ \&isAllowed $=y$.

Bhandari, Mahabir, Erol Chartan, Elaine Hale, Bruce Hedman, Reid (Rusty) Heffner, Paul Lemar, Sachin Nimbalkar, et al. 2018. "Modeling the Impact of Flexible CHP on California's Future Electric Grid." Technical Report. U.S. Department of Energy. https://www.energy.gov/eere/amo/downloads/modeling-impact-flexible-chp-california-sfuture-electric-grid-january-2018.

Brooker, A., J. Gonder, S. Lopp, and J. Ward. 2015. "ADOPT: A Historically Validated Light Duty Vehicle Consumer Choice Model." In SAE Technical Paper 2015-01-0974. Detroit, Michigan: SAE International. http://www.nrel.gov/docs/fy15osti/63608.pdf.

Bush, B., M. Muratori, C. Hunter, J. Zuboy, and M. Melaina. 2017. "Scenario Evaluation and Regionalization Analysis (SERA) Model: Demand Side and Refueling Infrastructure Build-Out. Supporting Documentation for the H2USA National Scenario Report." Technical Report NREL/TP-5400-70090. Golden, Colorado: National Renewable Energy Laboratory.

Deru, Michael, Kristin Field, Daniel Studer, Kyle Benne, Brent Griffith, Paul Torcellini, Bing Liu, et al. 2011. "US Department of Energy Commercial Reference Building Models of the National Building Stock."

Goel, Supriya, M. Rosenberg, R. Athalye, Y. Xie, W. Wang, R. Hart, J. Zhang, and V. Mendon. 2014. "Enhancements to ASHRAE Standard 90.1 Prototype Building Models." Technical Report PNNL-23269. Pacific Northwest National Laboratory. https://www.energycodes.gov/sites/default/files/documents/PrototypeModelEnhancements_2014.pdf. 


\section{References, cont.}

Hale, Elaine, Henry Horsey, Brandon Johnson, Matteo Muratori, Eric Wilson, Brennan Borlaug, Craig Christensen, et al. 2018. "The Demand-Side Grid (dsgrid) Model Documentation." Technical Report NREL/TP-6A20-71492. Golden, Colorado: National Renewable Energy Laboratory (NREL). https://www.nrel.gov/docs/fy18osti/71492.pdf.

Hale, Elaine T., Brady L. Stoll, and Joshua E. Novacheck. 2018. “Integrating Solar into Florida's Power System: Potential Roles for Flexibility." Accepted by Solar Energy.

Roth, Amir, David Goldwasser, and Andrew Parker. 2016. "There's a Measure for That!" Energy and Buildings 117 (April): 321-31. https://doi.org/10.1016/j.enbuild.2015.09.056.

Stoll, Brady, Elizabeth Buechler, and Elaine Hale. 2017. "The Value of Demand Response in Florida." The Electricity Journal, Energy Policy Institute's Seventh Annual Energy Policy Research Conference, 30 (9): 57-64. https://doi.org/10.1016/j.tej.2017.10.004.

Wilson, Eric, Craig Christensen, Scott Horowitz, and Henry Horsey. 2016. "A High-Granularity Approach to Modeling Energy Consumption and Savings Potential in the U.S. Residential Building Stock." IBPSA-USA Journal 6 (1).

Wilson, Eric, Craig Christensen, Scott Horowitz, Joseph Robertson, and Jeff Maguire. 2017. "Electric End-Use Energy Efficiency Potential in the U.S. Single-Family Housing Stock." Technical Report NREL/TP-5500-65667. Golden, Colorado: National Renewable Energy Laboratory. https://www.nrel.gov/docs/fy17osti/65667.pdf.

Wood, Eric, Sesha Raghavan, Clement Rames, Joshua Eichman, and Marc Melaina. 2017. "Regional Charging Infrastructure for Plug-In Electric Vehicles: A Case Study of Massachusetts." National Renewable Energy Lab.(NREL), Golden, CO (United States). https://www.osti.gov/scitech/biblio/1339074. 


\section{Thank you}

\section{www.nrel.gov}

NREL/PR-6A20-72388

This work was authored by the National Renewable Energy Laboratory, operated by Alliance for Sustainable Energy, LLC, for the U.S. Department of Energy (DOE) under Contract No. DE-AC36-08GO28308. Funding provided by the U.S. Department of Energy Office of Energy Efficiency and Renewable Energy Office of Strategic Programs. The views expressed in the article do not necessarily represent the views of the DOE or the U.S. Government. The U.S. Government retains and the publisher, by accepting the article for publication, acknowledges that the U.S.

Government retains a nonexclusive, paid-up, irrevocable, worldwide license to publish or reproduce the published form of this work, or allow others to do so, for U.S. Government purposes.

\section{ENREL \\ Transforming ENERGY}

